\title{
DIFFUSION THROUGH NON-LINEAR ORTHOTROPIC CYLINDERS
}

\author{
F. DAI and K. R. RAJAGOPAL \\ Department of Mechanical Engineering, University of Pittsburgh, Pittsburgh, PA 15261, U.S.A.

\section{A. S. WINEMAN} \\ Department of Mechanical Engineering, University of Michigan, Ann Arbor, MI 48109, U.S.A.
}

\begin{abstract}
In this paper, we study the problems of radial diffusion of a fluid through a hollow non-linearly elastic orthotropic cylinder. It is found that shearing and stretching have qualitatively different effects on the diffusion process. The effect of orthotropy on the diffusion process is also studied. These results are compared with those for the isotropic case [cf. Int. J. Engng Sci. 25, 1441-1457 (1987)].
\end{abstract}

\section{INTRODUCTION}

The theory of interacting continua (cf. Truesdell [1], Bowen [2], Atkin and Craine [3], Bedford and Drumheller [4], Passman et al. [5]) has relevance to several important technical problems in biomechanics, dyeing of textiles, and diffusion through composites, polymers and wood products, filtration, and the flow of fluid-solid mixtures to name just a few. Despite such wide applicability, until recently few boundary value problems have been solved within the context of this theory. This void can be traced to the difficulties associated with specifying adequate number of boundary conditions for traction boundary value problems. The theory supposes the existence of partial stress tensors for each constituent making up the mixture. Thus, in the case of a mixture of two constituents, there would be a partial stress tensor and thus a partial traction vector associated with each constituent, acting on the boundary. While the total traction on the boundary is known, we do not know how this splits into the individual partial tractions.

This knowledge is essential if we are to solve the boundary value problem as it is defined by balance of linear momentum equation for each constituent. Recently, motivated by some earlier work (cf. Shi et al. [6], Rajagopal et al. [7]), Rajagopal et al. [8] have developed a method for developing additional boundary conditions for a class of problems, based on a thermodynamic principle. Using the above additional boundary condition, Gandhi et al. [9] solved several prototype problems involving a mixture of an isotropic non-linearly elastic solid and a fluid.

To date, boundary value problems within the context of the theory of interacting continua associated with the diffusion of a fluid through a non-linearly elastic solid have assumed the solid to be isotropic. However, in many applications, the solid is anisotropic. For example, biological materials are orthotropic or transversely isotropic. The method used by Gandhi et al. [9] can once again be used to generate additional boundary conditions for problems involving the diffusion of fluids through anisotropic non-linearly elastic solids.

In this paper, we study the radial diffusion of a fluid through an orthotropic non-linearly elastic cylinder. We assume a particular form for the specific Helmholtz free energy function appropriate for orthotropic solids. We investigate the effect of the anisotropy on the diffusion process. We also study how shearing and stretching the cylinder affect the diffusion. In Section 2 , the notations and the basic equations of the theory of interacting continua are introduced. The constitutive equations for the incompressible solid and the fluid are postulated and the boundary conditions are developed. In the next section, the appropriate specific Helmholtz free energy function is introduced. In Section 4, the diffusion of a fluid through a hollow non-linearly elastic orthotropic cylinder is studied in detail. Section 5 is devoted to a discussion of the diffusion of a fluid through a non-linearly elastic orthotropic cylinder that is both sheared and stretched. The effect of the shearing and stretching are delineated. 


\section{PRELIMINARIES: NOTATIONS AND BASIC EQUATIONS}

We consider the interaction of two continua, a solid $S_{1}$ and a fluid $S_{2}$. At any time $t$, it is assumed that each place in the region of space is occupied by particles belonging to both $S_{1}$ and $S_{2}$. Let $\mathbf{X}$ and $\mathbf{Y}$ denote reference positions of typical particles of $S_{1}$ and $S_{2}$. The motions of $S_{1}$ and $S_{2}$ are denoted, respectively, by

$$
\mathbf{x}=\chi_{1}(\mathbf{X}, t) \text { and } \mathbf{y}=\chi_{2}(\mathbf{Y}, t)
$$

We shall assume that these motions are one-to-one, continuous and invertible. The various kinematical quantities associated with the solid $S_{1}$ and the fluid $S_{2}$ can be defined in the usual manner, i.e.

\begin{tabular}{lcc} 
Velocity & \multicolumn{1}{c}{$S_{1}$} & $S_{2}$ \\
Acceleration & $\mathbf{u}=\frac{\mathrm{d} \chi_{1}}{\mathrm{~d} t}$, & $\mathbf{w}=\frac{\mathrm{d} \boldsymbol{\chi}_{2}}{\mathrm{~d} t}$, \\
Velocity gradient & $\boldsymbol{a}_{s}=\frac{\mathrm{d}^{2} \boldsymbol{\chi}_{1}}{\mathrm{~d} t^{2}}$, & $\mathbf{a}_{f}=\frac{\mathrm{d}^{2} \chi_{2}}{\mathrm{~d} t^{2},}$ \\
Stretching & $\mathbf{L}=\frac{\partial \mathbf{u}}{\partial \mathbf{x}}$, & $\mathbf{M}=\frac{\partial \mathbf{w}}{\partial \mathbf{y}}$, \\
Vorticity & $\mathbf{d}=\frac{1}{2}\left(\mathbf{L}+\mathbf{L}^{T}\right)$, & $\mathbf{f}=\frac{1}{2}\left(\mathbf{M}+\mathbf{M}^{T}\right)$, \\
& $\mathbf{T}=\frac{1}{2}\left(\mathbf{L}-\mathbf{L}^{T}\right)$, & $\mathbf{\Lambda}=\frac{1}{2}\left(\mathbf{M}-\mathbf{M}^{T}\right)$.
\end{tabular}

The deformation gradient associated with $S_{1}$ is given by

$$
\mathbf{F}=\frac{\partial \mathbf{x}_{1}}{\partial \mathbf{X}} \text {. }
$$

Next, we document the basic equations of the theory of interacting continua.

\subsection{Conservation of mass}

Let $\rho_{1}$ and $\rho_{2}$ denote the mass densities of the solid and fluid, respectively. The total density $\rho$ of the mixture is given by

$$
\rho=\rho_{1}+\rho_{2} .
$$

The conservation of mass for the solid $S_{1}$ is given by

$$
\rho_{1}|\operatorname{det} \mathbf{F}|=\rho_{10}
$$

and for the fluid $S_{2}$ by

$$
\frac{\partial \rho_{2}}{\partial t}+\operatorname{div}\left(\rho_{2} \mathbf{w}\right)=0
$$

\subsection{Conservation of linear momentum}

Let $\sigma$ and $\pi$ denote the partial stress tensors associated with the solid and fluid, respectively. The total stress tensor is defined by

$$
\mathbf{T}=\boldsymbol{\sigma}+\boldsymbol{\pi}
$$

It is assumed that there is no external body force. The mechanical interaction between the solid and fluid is accounted for by a diffusive body force $b$. Then, the balances of linear momentum 
for the solid and fluid are, respectively

and

$$
\operatorname{div} \boldsymbol{\sigma}-\mathbf{b}=\rho_{1} \mathbf{a}_{s},
$$

$$
\operatorname{div} \boldsymbol{\pi}+\mathbf{b}=\rho_{2} \mathbf{a}_{f} .
$$

\subsection{Conservation of angular momentum}

The conservation of angular momentum for a mixture states that the total stress is symmetric, i.e.

$$
\sigma+\pi=\sigma^{T}+\pi^{T} .
$$

However, the partial stresses need not be symmetric.

\subsection{Surface conditions}

Let $\mathbf{n}, \mathbf{s}$ and $\mathbf{p}$ denote the unit outer normal vector at a point on the surface of the mixture region, the partial traction vectors taken by $S_{1}$ and $S_{2}$, respectively. Then

$$
\mathbf{s}=\sigma^{T} \mathbf{n} \text { and } \mathbf{p}=\pi^{T} \mathbf{n} .
$$

\subsection{Thermodynamical considerations}

Let the Helmboltz free energy per unit mass of $S_{1}$ and $S_{2}$ be denoted by $A_{1}$ and $A_{2}$, respectively. The Helmboltz free energy per unit mass of the mixture is defined by

Note that by setting

$$
\rho A=\rho_{1} A_{1}+\rho_{2} A_{2} .
$$

$$
\begin{gathered}
\mathbf{b}=\operatorname{grad} \phi_{1}+\overline{\mathbf{b}}=-\operatorname{grad} \phi_{2}+\overline{\mathbf{b}}, \\
\mathbf{\sigma}=\phi_{1} \mathbf{I}+\overline{\mathbf{\sigma}}, \\
\boldsymbol{\pi}=\phi_{2} \mathbf{I}+\overline{\boldsymbol{\pi}},
\end{gathered}
$$

where

$$
\phi_{1}=\rho_{1}\left(A_{1}-A\right), \quad \phi_{2}=\rho_{2}\left(A_{2}-A\right), \quad \phi_{1}+\phi_{2}=0 .
$$

Equations (7)-(10) become

$$
\begin{gathered}
\mathbf{T}=\overline{\mathbf{\sigma}}+\overline{\boldsymbol{\pi}}, \\
\operatorname{div} \overline{\boldsymbol{\sigma}}-\overline{\mathbf{b}}=\rho_{1} \mathbf{a}_{s}, \\
\operatorname{div} \overline{\boldsymbol{\pi}}+\overline{\mathbf{b}}=\rho_{2} \mathbf{a}_{f}, \\
\overline{\mathbf{\sigma}}+\overline{\boldsymbol{\pi}}=\overline{\boldsymbol{\sigma}}^{T}+\overline{\boldsymbol{\pi}}^{T},
\end{gathered}
$$

But, the terms in $\sigma, \pi$ and $b$ which depend on $\phi_{1}$ and $\phi_{2}$ do not contribute to the equations of motion or total stress (cf. Green and Naghdi [10]).

\subsection{Volume additivity constraint}

We restrict our attention to a mixture of incompressible materials. This implies that the motion of the interacting continua is such that it meets the following volume additivity constraint (cf. Mills [11]):

$$
\frac{\rho_{1}}{\rho_{10}}+\frac{\rho_{2}}{\rho_{20}}=1
$$

\subsection{Entropy inequality equation}

We refer the reader to [6] for a more detailed discussion of the entropy inequality. The stress is split into a static and dynamic part (cf. [6]). By using standard thermodynamic arguments, it can be shown that the equation used to restrict the constitutive equations for the dynamic part of the partial stress and the diffusive force is

$$
\bar{\sigma}_{(k i)}^{d} d_{i k}+\bar{\pi}_{(k i)}^{d} f_{i k}+\bar{\sigma}_{[k i]}^{d}\left(\Gamma_{i k}-\Lambda_{i k}\right)+\bar{b}_{k}^{d}\left(u_{k}-w_{k}\right)-\frac{q^{*}}{\theta} \frac{\partial \theta}{\partial x_{k}} \geq 0 .
$$




\subsection{Constitutive equations}

We assume that the mixture consists of an orthotropic elastic solid and a fluid. Accordingly, all constitutive functions are assumed to depend on the following variables (cf. [6]):

$$
\mathbf{F}, \nabla \mathbf{F}, \rho_{2}, \operatorname{grad} \rho_{2}, \theta, \operatorname{grad} \theta, \mathbf{u}, \mathbf{w}, \mathbf{L} \text { and } \mathbf{M},
$$

where $\theta$ denotes the absolute temperature. A lengthy but standard argument, based on the entropy production inequality and restrictions due to frame indifference and material symmetry lead to Helmholtz free energy per unit mass of the mixture to have the following form (cf. Green and Adkins [12]):

$$
\hat{A}=A\left(E_{11}, E_{22}, E_{33}, E_{12}^{2}, E_{13}^{2}, E_{23}^{2}, I_{3}, \rho_{2}, \theta\right),
$$

where

$$
E_{i j}=\frac{1}{2}\left(F_{i j} F_{l j}-\delta_{i j}\right)
$$

It follows from (5) and (21) that

$$
I_{3}=|\operatorname{det} \mathbf{F}|=\left(1-\frac{\rho_{2}}{\rho_{20}}\right)^{-1}
$$

We shall restrict our attention to isothermal problems. By virtue of (26), (24) can be expressed as

$$
A=A\left(E_{11}, E_{22}, E_{33}, E_{12}^{2}, E_{13}^{2}, E_{23}^{2}, \rho_{2}\right) .
$$

The constitutive relations for partial stresses and diffusive body force have the form (cf. [6])

$$
\begin{gathered}
\bar{\sigma}_{k i}=\bar{\sigma}_{k i}^{s}+\bar{\sigma}_{k i}^{d}=\rho \frac{\partial A}{\partial F_{i j}} F_{k j}-p \frac{\rho_{1}}{\rho_{10}} \delta_{k i}+\bar{\sigma}_{k i}^{d}, \\
\bar{\pi}_{k i}=\bar{\pi}_{k i}^{s}+\bar{\pi}_{k i}^{d}=-\rho_{2} \rho \frac{\partial A}{\partial \rho_{2}} \delta_{k i}-p \frac{\rho_{2}}{\rho_{20}} \delta_{k i}+\bar{\pi}_{k i}^{d},
\end{gathered}
$$

and

$$
\bar{b}_{k}=\bar{b}_{k}^{s}+\bar{b}_{k}^{d}=-\rho_{2} \frac{\partial F_{i j}}{\partial x_{k}} \frac{\partial A}{\partial F_{i j}}+\rho_{1} \frac{\partial A}{\partial \rho_{2}} \frac{\partial \rho_{2}}{\partial x_{k}}-\frac{p}{\rho_{10}} \frac{\partial \rho_{1}}{\partial x_{k}}+\bar{b}_{k}^{d},
$$

We shall assume that the dynamical parts of the partial stresses and diffusive force depend on the dynamic terms and are given by

and

$$
\begin{aligned}
& \bar{\sigma}_{k i}^{d}=\bar{\sigma}_{k i}^{d}\left(d_{m n}, f_{m n}\right), \\
& \bar{\pi}_{k i}^{d}=\bar{\pi}_{k i}^{d}\left(d_{m n}, f_{m n}\right),
\end{aligned}
$$

$$
\bar{b}_{k}^{d}=\bar{b}_{k}^{d}\left(u_{m}-w_{m}\right)
$$

Since the velocity of the fluid is always very small, we assume that the stresses depend on the dynamic variables linearly. We also assume that all the constitutive equations are independent of the rate of deformation tensor of the solid. Notice that

and

$$
\frac{\partial A}{\partial F_{i j}} F_{k i}=\frac{1}{2} \frac{\partial A}{\partial E_{p q}}\left(F_{i q} F_{k p}+F_{i p} F_{k q}\right)
$$

$$
\frac{\partial F_{i j}}{\partial x_{k}} \frac{\partial A}{\partial F_{i j}}=\frac{1}{2} F_{i q} \frac{\partial F_{i l}}{\partial x_{k}}\left(\frac{\partial A}{\partial E_{l q}}+\frac{\partial A}{\partial E_{q l}}\right)
$$

It follows, from the dissipation inequality and the assumption that the stresses depend linearly 
on the dynamic variables, that (cf. Dai [13])

$$
\begin{gathered}
\bar{\sigma}_{k i}=-p \frac{\rho_{1}}{\rho_{10}} \delta_{k i}+\rho\left[F_{i 1} F_{k 1} \frac{\partial A}{\partial E_{11}}+F_{i 2} F_{k 2} \frac{\partial A}{\partial E_{22}}+F_{i 3} F_{k 3} \frac{\partial A}{\partial E_{33}}\right. \\
+\left(F_{i 2} F_{k 1}+F_{i 1} F_{k 2}\right) E_{12} \frac{\partial A}{\partial E_{12}^{2}}+\left(F_{i 3} F_{k 1}+F_{i 1} F_{k 3}\right) E_{13} \frac{\partial A}{\partial E_{13}^{2}} \\
\left.+\left(F_{i 3} F_{k 2}+F_{i 2} F_{k 3}\right) E_{23} \frac{\partial A}{\partial E_{23}^{2}}\right], \\
\bar{b}_{k}=-p \frac{\partial}{\partial x_{k}}\left(\frac{\rho_{1}}{\rho_{10}}\right)+\rho_{1} \frac{\partial A}{\partial \rho_{2}} \frac{\partial \rho_{2}}{\partial x_{k}}+\alpha \frac{\rho_{1} \rho_{2}}{\rho_{10} \rho_{20}}\left(\delta_{k i}-w_{k}\right) \\
-\frac{1}{2} \rho_{2} F_{i q} \frac{\partial F_{i l}}{\partial x_{k}}\left(\frac{\partial A}{\partial E_{l q}}+\frac{\partial A}{\partial E_{q l}}\right),
\end{gathered}
$$

where

$$
\alpha \frac{\rho_{1} \rho_{2}}{\rho_{10} \rho_{20}}=c .
$$

and we have neglected the viscous effects in the fluid, since we are interested in the diffusion of an ideal fluid. It is also useful to record the representation for the total stress

$$
\begin{aligned}
T_{k i}= & \bar{\sigma}_{k i}+\bar{\pi}_{k i} \\
= & -p \delta_{k i}-\rho \rho_{2} \frac{\partial A}{\partial \rho_{2}} \delta_{k i}+\rho\left[F_{i 1} F_{k 1} \frac{\partial A}{\partial E_{11}}+F_{i 2} F_{k 2} \frac{\partial A}{\partial E_{22}}+F_{i 3} F_{k 3} \frac{\partial A}{\partial E_{33}}\right. \\
& +\left(F_{i 2} F_{k 1}+F_{i 1} F_{k 2}\right) E_{12} \frac{\partial A}{\partial E_{12}^{2}}+\left(F_{i 3} F_{k 1}+F_{i 1} F_{k 3}\right) E_{13} \frac{\partial A}{\partial E_{13}^{2}} \\
& \left.+\left(F_{i 3} F_{k 2}+F_{i 2} F_{k 3}\right) E_{23} \frac{\partial A}{\partial E_{23}^{2}}\right] .
\end{aligned}
$$

In the remainder of this paper, we shall only need to use $\bar{\sigma}, \bar{\pi}$ and $\bar{b}$. For notational convenience, we now drop the superposed bars.

\subsection{Boundary conditions}

A difficulty with the theory of mixtures is that there is no clear physical guidance for specifying partial tractions. Only the total surface traction $\mathbf{s}+\mathbf{p}$ can be specified, and this in general is not sufficient to solve boundary value problems. Rajagopal et al. [8] have suggested a method for generating appropriate additional boundary conditions for a class of boundary value problems. This method of generating additional boundary conditions is based on the assumption that the boundary of the solid-fluid mixture is in a saturated state.

In such a state we shall assume that the variation in the Helmholtz free energy equals the variation in the work due to the virtual displacement (cf. [8]). Thus,

$$
\delta W=m \delta A+A \delta m,
$$

where $\delta m, \delta A$ and $\delta W$ denote the variation in the mass, specific Helmholtz free energy and the work done due to the virtual deformation. Since

it follows that

$$
m=\rho \operatorname{det} \mathbf{F}=\rho_{20} \operatorname{det} \mathbf{F}+\left(\rho_{10}-\rho_{20}\right),
$$

$$
\delta m=\rho_{20} \delta(\operatorname{det} \mathbf{F})
$$


Also,

$$
\begin{aligned}
\delta A= & \frac{\partial A}{\partial E_{11}} \delta E_{11}+\frac{\partial A}{\partial E_{22}} \delta E_{22}+\frac{\partial A}{\partial E_{33}} \delta E_{33}+2 E_{12} \frac{\partial A}{\partial E_{12}^{2}} \delta E_{12} \\
& +2 E_{13} \frac{\partial A}{\partial E_{13}^{2}} \delta E_{13}+2 E_{23} \frac{\partial A}{\partial E_{23}^{2}} \delta E_{23}+\frac{\partial A}{\partial \rho_{2}} \delta \rho_{2} .
\end{aligned}
$$

Depending on the specific problem under consideration, (41) leads to a relationship between the strain components and the stresses $T_{11}, T_{22}, T_{33}$ etc. We shall derive the boundary condition appropriate to the specific boundary value problem under consideration in due course.

\section{SPECIFIC HELMHOLTZ FREE ENERGY FUNCTION}

In order to evaluate the partial stresses and diffusive forces, we should specify the free energy function for the mixture. We already have the general form of the free energy $A=A\left(E_{11}, E_{22}, E_{33}, E_{12}^{2}, E_{13}^{2}, E_{23}^{2}, \rho_{2}\right)$. We shall assume that

$$
\begin{aligned}
A= & K_{1} E_{11}+K_{2} E_{22}+K_{3} E_{33}+K_{4} E_{11} E_{22}+K_{5} E_{11} E_{33}+K_{6} E_{22} E_{33} \\
& +K_{7} E_{11}^{2}+K_{8} E_{22}^{2}+K_{9} E_{33}^{2}+K_{10} E_{12}^{2}+K_{11} E_{13}^{2}+K_{12} E_{23}^{2} \\
& +\left(K_{13} E_{11}+K_{14} E_{22}+K_{15} E_{33}\right) \frac{\rho_{2}}{\rho_{20}}+K f\left(\frac{\rho_{2}}{\rho_{20}}\right)
\end{aligned}
$$

where $K_{1}, K_{2}, \ldots, K_{15}$ and $K$ are constants and $f$ is a function of $\rho_{2} / \rho_{20}$. When $K_{1}=K_{2}=K_{3}$ and $K_{n}=0, n=4,5, \ldots 15$, we recover the isotropic model. Its specific form should be determined by experiment for a specific material. To make comparison with previous work in this area (cf. [6], [7] and [9]), we use

where

$$
f\left(\frac{\rho_{2}}{\rho_{20}}\right)=\frac{1-v}{v} \ln (1-v)+\chi(1-v),
$$

$$
v=(\operatorname{det} \mathbf{F})^{-1}=1-\frac{\rho_{2}}{\rho_{20}}
$$

which is appropriate for a mixture of a rubber-like solid and a solvent. Then, we get the final form of the free energy in our investigation:

$$
\begin{aligned}
A= & K_{1} E_{11}+K_{2} E_{22}+K_{3} E_{33}+K_{4} E_{11} E_{22}+K_{5} E_{11} E_{33}+K_{6} E_{22} E_{33} \\
& +K_{7} E_{11}^{2}+K_{8} E_{22}^{2}+K_{9} E_{33}^{2}+K_{10} E_{12}^{2}+K_{11} E_{13}^{2}+K_{12} E_{23}^{2} \\
& +\left(K_{13} E_{11}+K_{14} E_{22}+K_{15} E_{33}\right) \frac{\rho_{2}}{\rho_{20}}+K\left[\frac{1-v}{v} \ln (1-v)+\chi(1-v)\right]
\end{aligned}
$$

\section{DIFFUSION OF A FLUID THROUGH A HOLLOW ELASTIC ORTHOTROPIC CYLINDER}

Consider a hollow elastic orthotropic cylinder that has been axially stretched and radially expanded so that its outer surface in the deformed state is rigidly bonded to the inner surface of a rigid hollow cylindrical porous support (See Fig. 1). Suppose that the fluid diffuses in a radial

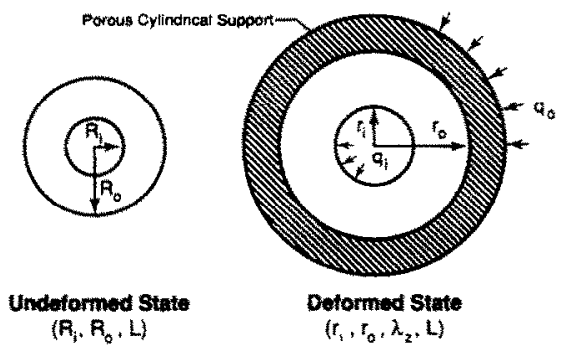

Fig. 1. Diffusion through a hollow cylinder. 
direction. As the solid absorbs the fluid and swells, the outer surface of the elastic cylinder remains fixed while the inner surface changes position. We consider the situation in which the solid cylinder has reached a fixed state of deformation and the fluid velocity field is steady.

For the problem under consideration, we shall assume an axially symmetric deformation of the form in a cylindrical coordinate system:

$$
\begin{gathered}
r=r(R), \\
\theta=\Theta, \\
z=\lambda Z,
\end{gathered}
$$

where $\lambda$ is assumed to be constant. The stretch ratios in the radial, circumferential and axial direction are defined, respectively, through

$$
\lambda_{r}=\frac{\mathrm{d} r}{\mathrm{~d} R}, \quad \lambda_{\theta}=\frac{r}{R}, \quad \lambda_{z}=\frac{\mathrm{d} z}{\mathrm{~d} Z}=\lambda .
$$

We shall assume the velocity field to be of the form

$$
w_{r}=w(r) \text { and } w_{\theta}=w_{z}=0 .
$$

It follows from (49)-(52) that the deformation gradient for the solid is given by

$$
\mathbf{F}=\left[\begin{array}{ccc}
\lambda_{r} & 0 & 0 \\
0 & \lambda_{\theta} & 0 \\
0 & 0 & \lambda
\end{array}\right]
$$

Thus, the Green-St. Venant strain tensor $\mathbf{E}$ is given by

$$
\mathbf{E}=\frac{1}{2}\left(\mathbf{F}^{T} \mathbf{F}-\mathbf{I}\right)=\frac{1}{2}\left[\begin{array}{ccc}
\lambda_{r}^{2}-1 & 0 & 0 \\
0 & \lambda_{\theta}^{2}-1 & 0 \\
0 & 0 & \lambda^{2}-1
\end{array}\right]
$$

The only non-zero physical components of the gradient of the deformation gradient are

$$
F_{r r ; r}=\frac{\mathrm{d} \lambda_{r}}{\mathrm{~d} r}, \quad F_{\theta \theta ; r}=\frac{\mathrm{d} \lambda_{\theta}}{\mathrm{d} r}, \quad F_{\theta r ; \theta}=F_{r \theta ; \theta}=\frac{1}{r}\left(\lambda_{r}-\lambda_{\theta}\right) .
$$

The conservation of mass for the fluid, (6), reduces to

which can be integrated to yield

$$
\frac{1}{r} \frac{\mathrm{d}}{\mathrm{d} r}\left(r \rho_{2} w\right)=0
$$

$$
r \rho_{2} w=F,
$$

where the constant $F$ denotes the mass flux through a unit length of the cylinder in unit time. Equation (58) states that the mass flux is the same through all surfaces $r=$ constant.

From the constitutive equations (36)-(38) and the assumed form for the specific Helmholtz free energy (48), it follows that the partial stresses for the solid are given by

$$
\begin{aligned}
& \sigma_{r r}=-\rho \frac{\rho_{1}}{\rho_{10}}+\frac{1}{2} \rho \lambda_{r}^{2}\left[2 K_{1}+K_{4}\left(\lambda_{\theta}^{2}-1\right)+K_{5}\left(\lambda^{2}-1\right)+2 K_{7}\left(\lambda_{r}^{2}-1\right)+2 K_{13} \frac{\rho_{2}}{\rho_{20}}\right] \\
& \sigma_{\theta \theta}=-p \frac{\rho_{1}}{\rho_{10}}+\frac{1}{2} \rho \lambda_{\theta}^{2}\left[2 K_{2}+K_{4}\left(\lambda_{r}^{2}-1\right)+K_{6}\left(\lambda^{2}-1\right)+2 K_{8}\left(\lambda_{\theta}^{2}-1\right)+2 K_{14} \frac{\rho_{2}}{\rho_{20}}\right] \\
& \sigma_{z z}=-p \frac{\rho_{1}}{\rho_{10}}+\frac{1}{2} \rho \lambda^{2}\left[2 K_{3}+K_{5}\left(\lambda_{r}^{2}-1\right)+K_{6}\left(\lambda_{\theta}^{2}-1\right)+2 K_{9}\left(\lambda^{2}-1\right)+2 K_{15} \frac{\rho_{2}}{\rho_{20}}\right]
\end{aligned}
$$

and

$$
\sigma_{\theta z}=\sigma_{r z}=\sigma_{r \theta}=0
$$


The partial stresses for the fluid are

$$
\pi_{r r}=\pi_{\theta \theta}=\pi_{2 z}=-p \frac{\rho_{2}}{\rho_{20}}+\frac{1}{2} \rho\left[K_{13}\left(\lambda_{r}^{2}-1\right)+K_{14}\left(\lambda_{\theta}^{2}-1\right)+K_{15}\left(\lambda^{2}-1\right)+2 K f^{\prime}\right] \frac{\rho_{2}}{\rho_{20}},
$$

and

where

$$
\pi_{\theta z}=\pi_{r z}=\pi_{r \theta}=0
$$

$$
f^{\prime}\left(\frac{\rho_{2}}{\rho_{20}}\right)=\frac{1}{v^{2}}\left[\ln (1-v)+v+\chi v^{2}\right]
$$

The diffusive body force components are given by

$$
\begin{aligned}
b_{r}= & -p \frac{\mathrm{d}}{\mathrm{d} r}\left(\frac{\rho_{1}}{\rho_{10}}\right)-\alpha \frac{\rho_{1} \rho_{2}}{\rho_{10} \rho_{20}} w+\frac{1}{2} \rho_{1}\left[K_{13}\left(\lambda_{r}^{2}-1\right)\right. \\
& \left.+K_{14}\left(\lambda_{\theta}^{2}-1\right)+K_{15}\left(\lambda^{2}-1\right)+2 K f^{\prime}\right] \frac{\mathrm{d}}{\mathrm{d} r}\left(\frac{\rho_{2}}{\rho_{20}}\right) \\
& -\frac{1}{2} \rho_{2}\left\{\lambda_{r}\left[2 K_{1}+K_{4}\left(\lambda_{\theta}^{2}-1\right)+K_{5}\left(\lambda^{2}-1\right)+2 K_{7}\left(\lambda_{r}^{2}-1\right)+2 K_{13} \frac{\rho_{2}}{\rho_{20}}\right] \frac{\mathrm{d} \lambda_{r}}{\mathrm{~d} r}\right. \\
& \left.+\lambda_{\theta}\left[2 k_{2}+K_{4}\left(\lambda_{r}^{2}-1\right)+K_{6}\left(\lambda^{2}-1\right)+2 K_{8}\left(\lambda_{\theta}^{2}-1\right)+2 K_{14} \frac{\rho_{2}}{\rho_{20}}\right] \frac{\mathrm{d} \lambda_{\theta}}{\mathrm{d} r}\right\},
\end{aligned}
$$

and

$$
b_{\theta}=b_{z}=0 .
$$

By virtue of (40), the components of total traction are

$$
\begin{aligned}
T_{r r}= & -p-\frac{1}{2} \rho\left[K_{13}\left(\lambda_{r}^{2}-1\right)+K_{14}\left(\lambda_{\theta}^{2}-1\right)+K_{15}\left(\lambda^{2}-1\right)+2 K f^{\prime}\right] \frac{\rho_{2}}{\rho_{20}} \\
& +\frac{1}{2} \rho \lambda_{r}^{2}\left[2 K_{1}+K_{4}\left(\lambda_{\theta}^{2}-1\right)+K_{5}\left(\lambda^{2}-1\right)+2 K_{7}\left(\lambda_{r}^{2}-1\right)+2 K_{13} \frac{\rho_{2}}{\rho_{20}}\right] \\
T_{\theta \theta}= & -p-\frac{1}{2} \rho\left[K_{13}\left(\lambda_{r}^{2}-1\right)+K_{14}\left(\lambda_{\theta}^{2}-1\right)+K_{15}\left(\lambda^{2}-1\right)+2 K f^{\prime}\right] \frac{\rho_{2}}{\rho_{20}} \\
& +\frac{1}{2} \rho \lambda_{\theta}^{2}\left[2 K_{2}+K_{4}\left(\lambda_{r}^{2}-1\right)+K_{6}\left(\lambda^{2}-1\right)+2 K_{8}\left(\lambda_{\theta}^{2}-1\right)+2 K_{14} \frac{\rho_{2}}{\rho_{20}}\right], \\
T_{z z}= & -p-\frac{1}{2} \rho\left[K_{13}\left(\lambda_{r}^{2}-1\right)+K_{14}\left(\lambda_{\theta}^{2}-1\right)+K_{15}\left(\lambda^{2}-1\right)+2 K f^{\prime}\right] \frac{\rho_{2}}{\rho_{20}} \\
& +\frac{1}{2} \rho \lambda^{2}\left[2 K_{3}+K_{5}\left(\lambda_{r}^{2}-1\right)+K_{6}\left(\lambda_{\theta}^{2}-1\right)+2 K_{9}\left(\lambda^{2}-1\right)+2 K_{15} \frac{\rho_{2}}{\rho_{20}}\right],
\end{aligned}
$$

and

$$
T_{r \theta}=T_{r z}=T_{\theta z}=0 .
$$

For the problem under consideration, on neglecting the inertial effects, since the accelerations are small the balance of linear momentum for the solid reduces to

$$
\begin{gathered}
\frac{\partial \sigma_{r r}}{\partial r}+\frac{\sigma_{r r}-\sigma_{\theta \theta}}{r}-b_{r}=0, \\
\frac{1}{r} \frac{\partial \sigma_{\theta \theta}}{\partial \theta}=\frac{\partial \sigma_{z z}}{\partial z}=0 .
\end{gathered}
$$


It follows from (59)-(61), (66), (72) and (73) that

$$
\begin{aligned}
& -\left(\frac{\rho_{1}}{\rho_{10}}\right) \frac{\partial p}{\partial r}+\alpha \frac{\rho_{1} \rho_{2}}{\rho_{10} \rho_{20}} w-\frac{1}{2} \rho_{1}\left[K_{13}\left(\lambda_{r}^{2}-1\right)+K_{14}\left(\lambda_{\theta}^{2}-1\right)+K_{15}\left(\lambda^{2}-1\right)+2 K f^{\prime}\right] \frac{\mathrm{d}}{\mathrm{d} r}\left(\frac{\rho_{2}}{\rho_{20}}\right) \\
& +\frac{1}{2} \frac{\mathrm{d}}{\mathrm{d} r}\left\{\rho \lambda_{r}^{2}\left[2 K_{1}+K_{4}\left(\lambda_{\theta}^{2}-1\right)+K_{5}\left(\lambda^{2}-1\right)+K_{7}\left(\lambda_{r}^{2}-1\right)+2 K_{13} \frac{\rho_{2}}{\rho_{20}}\right]\right\} \\
& +\frac{1}{2} \frac{\rho}{r}\left\{\lambda_{r}^{2}\left[2 K_{1}+K_{4}\left(\lambda_{\theta}^{2}-1\right)+K_{5}\left(\lambda^{2}-1\right)+2 K_{7}\left(\lambda_{r}^{2}-1\right)+2 K_{13} \frac{\rho_{2}}{\rho_{20}}\right]\right. \\
& \left.-\lambda_{\theta}^{2}\left[2 K_{2}+K_{4}\left(\lambda_{r}^{2}-1\right)+K_{6}\left(\lambda^{2}-1\right)+2 K_{8}\left(\lambda_{\theta}^{2}-1\right)+2 K_{14} \frac{\rho_{2}}{\rho_{20}}\right]\right\} \\
& +\frac{1}{2} \rho_{2}\left\{\lambda_{r}\left[2 K_{1}+K_{4}\left(\lambda_{\theta}^{2}-1\right)+K_{5}\left(\lambda^{2}-1\right)+2 K_{7}\left(\lambda_{r}^{2}-1\right)+2 K_{13} \frac{\rho_{2}}{\rho_{20}}\right] \frac{\mathrm{d} \lambda_{r}}{\mathrm{~d} r}\right. \\
& \left.+\lambda_{\theta}\left[2 K_{2}+K_{4}\left(\lambda_{r}^{2}-1\right)+K_{6}\left(\lambda^{2}-1\right)+2 K_{8}\left(\lambda_{\theta}^{2}-1\right)+2 K_{14} \frac{\rho_{2}}{\rho_{20}}\right] \frac{\mathrm{d} \lambda_{\theta}}{\mathrm{d} r}\right\}=0,
\end{aligned}
$$

and

Equation (75) implies that

$$
-\frac{1}{r} \frac{\rho_{1}}{\rho_{10}} \frac{\partial p}{\partial \theta}=-\frac{\rho_{1}}{\rho_{10}} \frac{\partial p}{\partial z}=0
$$

$$
p=p(r) \text {. }
$$

Equation (74) gives one equation for the indeterminate scalar $p$, the radial deformation of the cylinder $r$ and the radial fluid velocity $w$.

Another appropriate equation is obtained by adding (8) and (9), using (7) and neglecting the inertia of the fluid. The balance of linear momentum reduces to

$$
\frac{\mathrm{d} T_{r r}}{\mathrm{~d} r}+\frac{T_{r r}-T_{\theta \theta}}{r}=0
$$

It follows from (68) and (69) that (77) becomes

$$
\begin{aligned}
& -\frac{\mathrm{d} p}{\mathrm{~d} r}-\frac{1}{2} \frac{\mathrm{d}}{\mathrm{d} r}\left\{\rho\left[K_{13}\left(\lambda_{r}^{2}-1\right)+K_{14}\left(\lambda_{\theta}^{2}-1\right)+K_{15}\left(\lambda^{2}-1\right)+2 K f^{\prime}\right] \frac{\rho_{2}}{\rho_{20}}\right\} \\
& +\frac{1}{2} \frac{\mathrm{d}}{\mathrm{d} r}\left\{\rho \lambda_{r}^{2}\left[2 K_{1}+K_{4}\left(\lambda_{\theta}^{2}-1\right)+K_{5}\left(\lambda^{2}-1\right)+2 K_{7}\left(\lambda_{r}^{2}-1\right)+2 K_{13} \frac{\rho_{2}}{\rho_{20}}\right]\right\} \\
& +\frac{\rho}{2 r}\left\{\lambda_{r}^{2}\left[2 K_{1}+K_{4}\left(\lambda_{\theta}^{2}-1\right)+K_{5}\left(\lambda^{2}-1\right)+2 K_{7}\left(\lambda_{r}^{2}-1\right)+2 K_{13} \frac{\rho_{2}}{\rho_{20}}\right]\right. \\
& \left.-\lambda_{\theta}^{2}\left[2 K_{2}+K_{4}\left(\lambda_{r}^{2}-1\right)+K_{6}\left(\lambda^{2}-1\right)+2 K_{8}\left(\lambda_{\theta}^{2}-1\right)+2 K_{14} \frac{\rho_{2}}{\rho_{20}}\right]\right\}=0 .
\end{aligned}
$$

From (74) and (78), we have

$$
\begin{aligned}
& \frac{1}{2} \frac{\rho_{1}}{\rho_{10}} \frac{\mathrm{d}}{\mathrm{d} r}\left\{\rho\left[K_{13}\left(\lambda_{r}^{2}-1\right)+K_{14}\left(\lambda_{\theta}^{2}-1\right)+K_{15}\left(\lambda^{2}-1\right)+2 K f^{\prime}\right] \frac{\rho_{2}}{\rho_{20}}\right\} \\
& -\frac{1}{2} \rho_{1}\left[K_{13}\left(\lambda_{r}^{2}-1\right)+K_{14}\left(\lambda_{\theta}^{2}-1\right)+K_{15}\left(\lambda^{2}-1\right)+2 K f^{\prime}\right] \frac{\mathrm{d}}{\mathrm{d} r}\left(\frac{\rho_{2}}{\rho_{20}}\right)+\alpha \frac{\rho_{1} \rho_{2}}{\rho_{10} \rho_{20}} w \\
& +\frac{1}{2} \frac{\rho_{2}}{\rho_{20}} \frac{\mathrm{d}}{\mathrm{d} r}\left\{\rho \lambda_{r}^{2}\left[2 K_{1}+K_{4}\left(\lambda_{\theta}^{2}-1\right)+K_{5}\left(\lambda^{2}-1\right)+2 K_{7}\left(\lambda_{r}^{2}-1\right)+2 K_{13} \frac{\rho_{2}}{\rho_{20}}\right]\right\} \\
& +\frac{\rho}{2 r} \frac{\rho_{2}}{\rho_{20}}\left\{\lambda_{r}^{2}\left[2 K_{1}+K_{4}\left(\lambda_{\theta}^{2}-1\right)+K_{5}\left(\lambda^{2}-1\right)+2 K_{7}\left(\lambda_{r}^{2}-1\right)+2 K_{13} \frac{\rho_{2}}{\rho_{20}}\right]\right. \\
& \left.-\lambda_{\theta}^{2}\left[2 K_{2}+K_{4}\left(\lambda_{r}^{2}-1\right)+K_{6}\left(\lambda^{2}-1\right)+2 K_{8}\left(\lambda_{\theta}^{2}-1\right)+2 K_{14} \frac{\rho_{2}}{\rho_{20}}\right]\right\}
\end{aligned}
$$




$$
\begin{aligned}
& +\frac{1}{2} \rho_{2}\left\{\lambda_{r}\left[2 K_{1}+K_{4}\left(\lambda_{\theta}^{2}-1\right)+K_{5}\left(\lambda^{2}-1\right)+2 K_{7}\left(\lambda_{r}^{2}-1\right)+2 K_{13} \frac{\rho_{2}}{\rho_{20}}\right] \frac{\mathrm{d} \lambda_{r}}{\mathrm{~d} r}\right. \\
& \left.+\lambda_{\theta}\left[2 K_{2}+K_{4}\left(\lambda_{r}^{2}-1\right)+K_{6}\left(\lambda^{2}-1\right)+2 K_{8}\left(\lambda_{\theta}^{2}-1\right)+2 K_{14} \frac{\rho_{2}}{\rho_{20}}\right] \frac{\mathrm{d} \lambda_{\theta}}{\mathrm{d} r}\right\}=0
\end{aligned}
$$

The fluid velocity $w$ can be eliminated in favor of the mass flux by using (58). Furthermore, it is preferable to regard all quantities as functions of the reference coordinate $R$. Thus, using $(52)_{1}$, we transform (79) to obtain

$$
\begin{aligned}
& \frac{1}{2} \frac{\rho_{1}}{\rho_{10}} \frac{\mathrm{d}}{\mathrm{d} R}\left\{\rho\left[K_{13}\left(\lambda_{r}^{2}-1\right)+K_{14}\left(\lambda_{\theta}^{2}-1\right)+K_{15}\left(\lambda^{2}-1\right)+2 K f^{\prime}\right] \frac{\rho_{2}}{\rho_{20}}\right\} \\
& -\frac{1}{2} \rho_{1}\left[K_{13}\left(\lambda_{r}^{2}-1\right)+K_{14}\left(\lambda_{\theta}^{2}-1\right)+K_{15}\left(\lambda^{2}-1\right)+2 K f^{\prime}\right] \frac{\mathrm{d}}{\mathrm{d} R}\left(\frac{\rho_{2}}{\rho_{20}}\right)+\alpha \frac{F \rho_{1} \lambda_{r}}{R \rho_{10} \rho_{20} \lambda_{\theta}} \\
& +\frac{1}{2} \frac{\rho_{2}}{\rho_{20}} \frac{\mathrm{d}}{\mathrm{d} R}\left\{\rho \lambda_{r}^{2}\left[2 K_{1}+K_{4}\left(\lambda_{\theta}^{2}-1\right)+K_{5}\left(\lambda^{2}-1\right)+2 K_{7}\left(\lambda_{r}^{2}-1\right)+2 K_{13} \frac{\rho_{2}}{\rho_{20}}\right]\right\} \\
& +\frac{\lambda_{r} \rho}{2 R \lambda_{\theta}} \frac{\rho_{2}}{\rho_{20}}\left\{\lambda_{r}^{2}\left[2 K_{1}+K_{4}\left(\lambda_{\theta}^{2}-1\right)+K_{5}\left(\lambda^{2}-1\right)+2 K_{7}\left(\lambda_{r}^{2}-1\right)+2 K_{13} \frac{\rho_{2}}{\rho_{20}}\right]\right. \\
& \left.-\lambda_{\theta}^{2}\left[2 K_{2}+K_{4}\left(\lambda_{r}^{2}-1\right)+K_{6}\left(\lambda^{2}-1\right)+2 K_{8}\left(\lambda_{\theta}^{2}-1\right)+2 K_{14} \frac{\rho_{2}}{\rho_{20}}\right]\right\} \\
& +\frac{1}{2} \rho_{2}\left\{\lambda_{r}\left[2 K_{1}+K_{4}\left(\lambda_{\theta}^{2}-1\right)+K_{5}\left(\lambda^{2}-1\right)+K_{7}\left(\lambda_{r}^{2}-1\right)+2 K_{13} \frac{\rho_{2}}{\rho_{20}}\right] \frac{\mathrm{d} \lambda_{r}}{\mathrm{~d} R}\right. \\
& \left.+\lambda_{\theta}\left[2 K_{2}+K_{4}\left(\lambda_{r}^{2}-1\right)+K_{6}\left(\lambda^{2}-1\right)+2 K_{8}\left(\lambda_{\theta}^{2}-1\right)+2 K_{14} \frac{\rho_{2}}{\rho_{20}}\right] \frac{\mathrm{d} \lambda_{\theta}}{\mathrm{d} R}\right\}=0 .
\end{aligned}
$$

We define the non-dimensional quantities

$$
\hat{R}=\frac{R}{R_{i}}, \quad \hat{\rho}_{1}=\frac{\rho_{1}}{\rho_{10}}, \quad \hat{\rho}_{2}=\frac{\rho_{2}}{\rho_{20}}, \quad \hat{\rho}=\frac{\rho}{\rho_{20}} .
$$

and introduce them into $(80)$ to obtain

$$
\begin{aligned}
& \frac{1}{2} \hat{\rho}_{1} \frac{\mathrm{d}}{\mathrm{d} \hat{R}}\left\{\hat{\rho} \hat{\rho}_{2}\left[\hat{K}_{13}\left(\lambda_{r}^{2}-1\right)+\hat{K}_{14}\left(\lambda_{\theta}^{2}-1\right)+\hat{K}_{15}\left(\lambda^{2}-1\right)+2 f^{\prime}\right]\right\} \\
& -\frac{1}{2} \hat{\rho}_{1}\left[\hat{K}_{13}\left(\lambda_{r}^{2}-1\right)+\hat{K}_{14}\left(\lambda_{\theta}^{2}-1\right)+\hat{K}_{15}\left(\lambda^{2}-1\right)+2 f^{\prime}\right] \frac{\rho_{10}}{\rho_{20}} \frac{\mathrm{d} \hat{\rho}_{2}}{\mathrm{~d} \hat{R}} \\
& +\frac{\hat{F}}{\lambda \hat{R} \lambda_{\theta}^{2}}+\frac{1}{2} \hat{\rho}_{2} \frac{\mathrm{d}}{\mathrm{d} \hat{R}}\left(\hat{\rho} \lambda_{r}^{2}\left[2 \hat{K}_{1}+\hat{K}_{4}\left(\lambda_{\theta}^{2}-1\right)+\hat{K}_{5}\left(\lambda^{2}-1\right)+2 \hat{K}_{7}\left(\lambda_{r}^{2}-1\right)+2 \hat{K}_{13} \hat{\rho}_{2}\right]\right\} \\
& +\frac{\hat{\rho}_{2} \hat{\rho} \lambda_{r}}{2 \hat{R} \lambda_{\theta}}\left\{\lambda_{r}^{2}\left[2 \hat{K}_{1}+\hat{K}_{4}\left(\lambda_{\theta}^{2}-1\right)+\hat{K}_{5}\left(\lambda^{2}-1\right)+2 \hat{K}_{7}\left(\lambda_{r}^{2}-1\right)+2 \hat{K}_{13} \hat{\rho}_{2}\right]\right. \\
& \left.-\lambda_{\theta}^{2}\left[2 \hat{K}_{2}+\hat{K}_{4}\left(\lambda_{r}^{2}-1\right)+\hat{K}_{6}\left(\lambda^{2}-1\right)+2 \hat{K}_{8}\left(\lambda_{\theta}^{2}-1\right)+2 \hat{K}_{14} \hat{\rho}_{2}\right]\right\} \\
& +\frac{1}{2} \hat{\rho}_{2}\left\{\lambda_{r}\left[2 \hat{K}_{1}+\hat{K}_{4}\left(\lambda_{\theta}^{2}-1\right)+\hat{K}_{5}\left(\lambda^{2}-1\right)+2 \hat{K}_{7}\left(\lambda_{r}^{2}-1\right)+2 \hat{K}_{13} \hat{\rho}_{2}\right] \frac{\mathrm{d} \lambda_{r}}{\mathrm{~d} \hat{R}}\right. \\
& \left.+\lambda_{\theta}\left[2 \hat{K}_{2}+\hat{K}_{4}\left(\lambda_{r}^{2}-1\right)+\hat{K}_{6}\left(\lambda^{2}-1\right)+2 \hat{K}_{8}\left(\lambda_{\theta}^{2}-1\right)+2 \hat{K}_{14} \hat{\rho}_{2}\right] \frac{\mathrm{d} \lambda_{\theta}}{\mathrm{d} \hat{R}}\right\}=0 .
\end{aligned}
$$

where

$$
\begin{aligned}
& \hat{K}_{1}=\frac{K_{1}}{K}, \quad \hat{K}_{2}=\frac{K_{2}}{K}, \quad \hat{K}_{4}=\frac{K_{4}}{K}, \quad \hat{K}_{5}=\frac{K_{5}}{K}, \\
& \hat{K}_{6}=\frac{K_{6}}{K}, \quad \hat{K}_{7}=\frac{K_{7}}{K}, \quad \hat{K}_{8}=\frac{K_{8}}{K}, \quad \hat{K}_{13}=\frac{K_{13}}{K}, \\
& \hat{K}_{14}=\frac{K_{14}}{K}, \quad \hat{K}_{15}=\frac{K_{15}}{K},
\end{aligned}
$$


and

$$
\hat{F}=\frac{\alpha F}{K \rho_{20}^{2}} .
$$

The second equation can be obtained from $(52)_{2}$. In nondimensional form, it is

$$
\frac{\mathrm{d} \lambda_{\theta}}{\mathrm{d} \hat{R}}=\frac{\lambda_{r}-\lambda_{\theta}}{\hat{R}}
$$

Now we turn to our attention of boundary conditions. In virtue of (44), for the problem under consideration, it follows that

$$
\delta A=\frac{\partial A}{\partial E_{r r}} \lambda_{r} \delta \lambda_{r}+\frac{\partial A}{\partial E_{\theta \theta}} \lambda_{\theta} \delta \lambda_{\theta}+\frac{\partial A}{\partial E_{z z}} \lambda_{z} \delta \lambda_{z}+\frac{\partial A}{\partial \rho_{2}} \delta \rho_{2} .
$$

From (43), (47) and (54), we have

$$
\begin{gathered}
\delta \rho_{2}=\rho_{20}\left[\frac{1}{\left(\lambda_{2} \lambda_{\theta} \lambda\right)^{2}}\left(\lambda_{\theta} \lambda \delta \lambda_{r}+\lambda_{r} \lambda \lambda_{\theta}+\lambda_{r} \lambda_{\theta} \delta \lambda\right)\right], \\
\delta m=\rho_{20}\left(\lambda_{\theta} \lambda \delta \lambda_{r}+\lambda_{r} \lambda \delta \lambda_{\theta}+\lambda_{r} \lambda_{\theta} \delta \lambda\right) .
\end{gathered}
$$

Also, it is easy to see that for the problem under consideration

$$
\delta W=T_{r r} \lambda_{\theta} \lambda \delta \lambda_{r}+T_{\theta \theta} \lambda_{r} \lambda \delta \lambda_{\theta}+T_{z z} \lambda_{r} \lambda_{\theta} \delta \lambda .
$$

It follows from (41)-(43) and (86)-(89) that

$$
\begin{aligned}
& T_{r r}=\rho_{20} A+\rho\left(\rho_{20}-\rho_{2}\right) \frac{\partial A}{\partial \rho_{2}}+\rho \lambda_{r}^{2} \frac{\partial A}{\partial E_{r r}}, \\
& T_{\theta \theta}=\rho_{20} A+\rho\left(\rho_{20}-\rho_{2}\right) \frac{\partial A}{\partial \rho_{2}}+\rho \lambda_{\theta}^{2} \frac{\partial A}{\partial E_{\theta \theta}}, \\
& T_{z z}=\rho_{20} A+\rho\left(\rho_{20}-\rho_{2}\right) \frac{\partial A}{\partial \rho_{2}}+\rho \lambda^{2} \frac{\partial A}{\partial E_{z z}} .
\end{aligned}
$$

Substituting (48) into (90) and considering the inner and outer surfaces of the cylinder, respectively, we have

and

$$
\begin{aligned}
-q_{i}= & \left\{\rho_{20} A+K \rho f^{\prime}\left(1-\frac{\rho_{2}}{\rho_{20}}\right)+\frac{1}{2} \rho \lambda_{r}^{2}\left[2 K_{1}+K_{4}\left(\lambda_{\theta}^{2}-1\right)\right.\right. \\
& \left.\left.+K_{5}\left(\lambda^{2}-1\right)+2 K_{7}\left(\lambda_{r}^{2}-1\right)+K_{13} \frac{\rho_{2}}{\rho_{29}}\right]\right\}\left.\right|_{\hat{k}=1},
\end{aligned}
$$

$$
\begin{aligned}
-q_{o}= & \left\{\rho_{20} A+K \rho f^{\prime}\left(1-\frac{\rho_{2}}{\rho_{20}}\right)+\frac{1}{2} \rho \lambda_{r}^{2}\left[2 K_{1}+K_{4}\left(\lambda_{\theta}^{2}-1\right)\right.\right. \\
& \left.\left.+K_{5}\left(\lambda^{2}-1\right)+2 K_{7}\left(\lambda_{r}^{2}-1\right)+K_{13} \frac{\rho_{2}}{\rho_{20}}\right]\right\}\left.\right|_{\hat{R}=a},
\end{aligned}
$$

where $a=R_{o} / R_{i}$ and $q_{i}$ and $q_{o}$ denote the pressures on the inner and outer surfaces of the cylindrical mixture region, respectively.

The numerical solution procedure is as follows. Since $r_{0}$ is specified, $\lambda_{\theta}(a)$ is known from $(52)_{2}$. For a specified value of $q_{0}, \lambda_{r}(a)$ can be solved from (94). The system (82) and (85) is then integrated from $\hat{R}=a$ to $\hat{R}=1$, giving $\lambda_{\theta}(1)$ and $\lambda_{r}(1)$. These values are then substituted into (93) to determine $q_{i}$. This gives the pressure difference $q_{i}-q_{o}$ for a specified value of mass flux $\hat{F}$. The quantities of interest can then be computed from the appropriate equations, e.g. $r=R \lambda_{\theta}(R)$ from $(52)_{2}$. An ordinary differential equation solver called DVERK from the International Mathematical and Statistical Library (IMSL) has been used to numerically integrate (82) and (85). This subroutine is based on Runge-Kutta-Verner method. The error tolerance of the numerical solution is of the order of $10^{-8}$. Some of the parameters used in our 


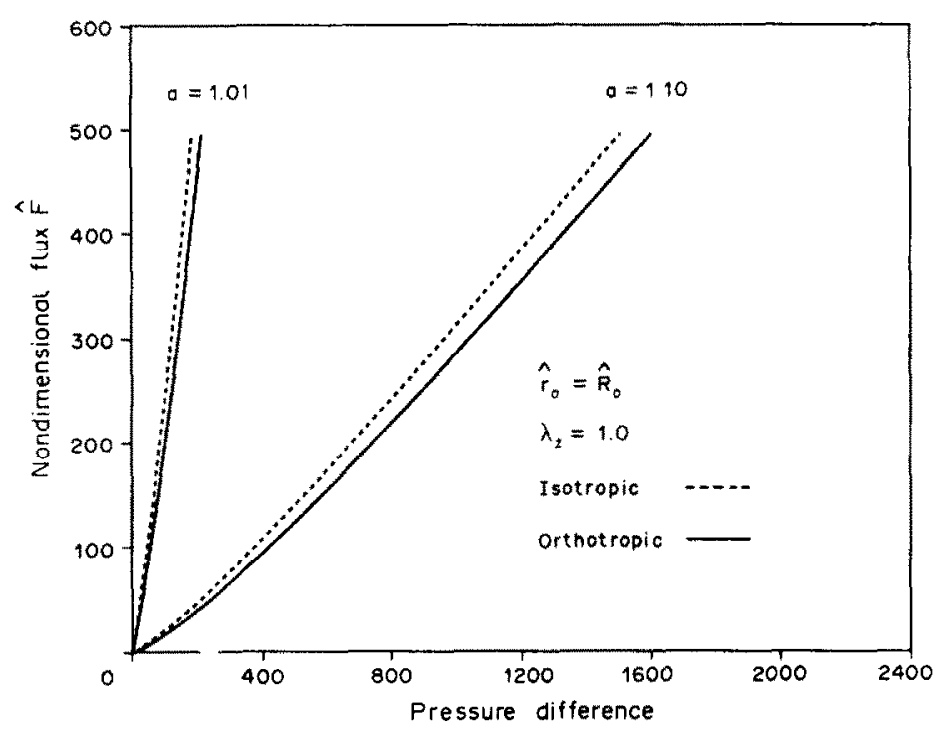

Fig. 2. Effect of the cylinder thickness on the flux for inward diffusion.

numerical simulation are the same as those used in [9] and [14]. Set

$$
\begin{gathered}
\rho_{10}=0.862 \mathrm{~g} / \mathrm{cc}=0.0311 \mathrm{lb} / \mathrm{in}^{3}, \\
\rho_{20}=0.869 \mathrm{~g} / \mathrm{cc}=0.0314 \mathrm{lb} / \mathrm{in}^{3}, \\
K_{3}=K_{4}=K_{5}=K_{6}=K_{9}=415, \\
K_{10}=K_{11}=K_{12}=K_{13}=K_{14}=K_{15}=0, \\
K=415, \\
\chi=0.5,
\end{gathered}
$$

Set $K_{1}=K_{2}=830, K_{7}=K_{8}=415$ for isotropic case and $K_{1}=K_{7}=1245, K_{2}=K_{8}=415$ for orthotropic case. The dimension of $K_{i}, i=1,2, \ldots, 15$, and $K$ is $\mathrm{in}^{2} / \mathrm{s}^{2}$.

We use the term "inward-diffusion" to mean the diffusion of a fluid through the cylinder from the outside to the inside and the term "outward-diffusion" to mean the diffusion from inside to the outside. The effects of deformations, elastic moduli and the structure of the Helmholtz free energy on fluxes have been studied for a number of different parameters for

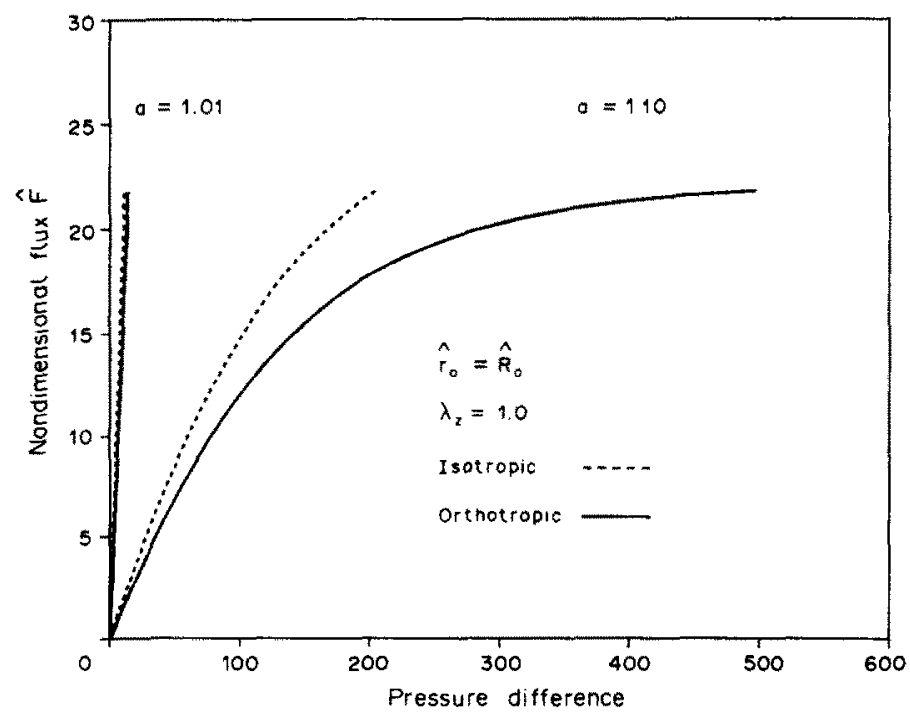

Fig. 3. Effect of the cylinder thickness on the flux for outward diffusion. 


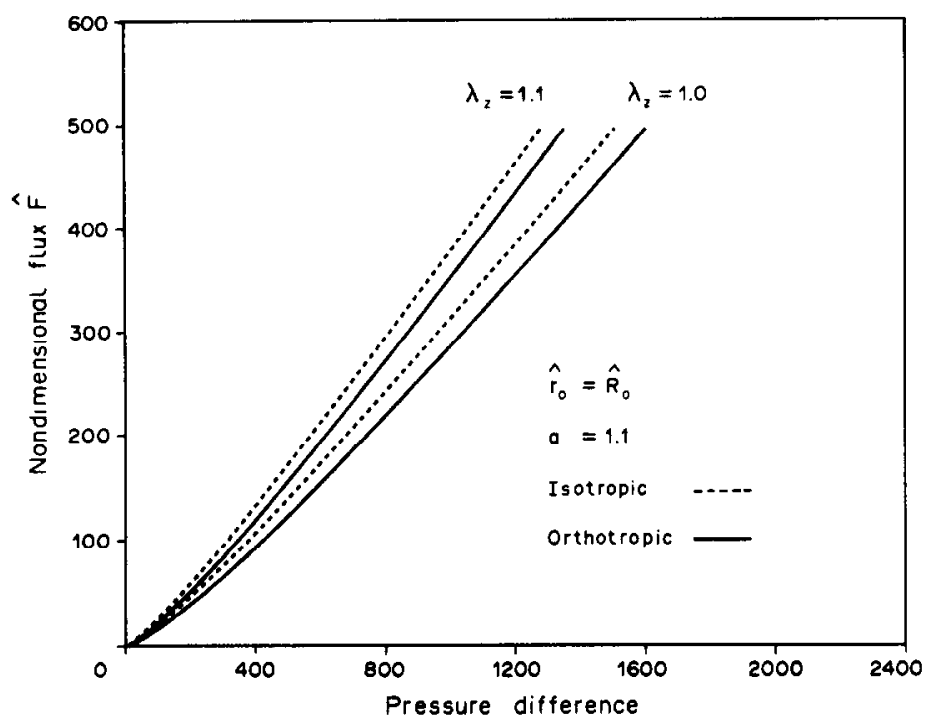

Fig. 4. Effect of the axial deformation on the flux for inward diffusion.

both the inward and outward-diffusions. The results for orthotropic case have been compared with those for isotropic case in figures.

\section{(i) Effect of deformation on flux}

Figures 2-7 show the influence of the cylinder thickness, the axial deformation and the radial expansion on flux. The results shown in Figs 2 and 3 are for the case in which there is no axial stretch and the cylinder is not radially expanded before the outer surface is bonded to the rigid porous support for the inward and outward-diffusions, respectively. It is found that the mass flux through a thin-walled cylinder is greater than that through a thick-walled cylinder for both inward and outward-diffusions. Figures 4 and 5 show the results for the case in which the axial stretch ratio is increased, and then the cylinder outer surface is restored to its original radius. This results in an increase in the mass flux. A similar increase occurs when there is no axial stretch, but the outer radius is increased before bonding, as shown in Figs 6 and 7 for the inward and outward-diffusions, respectively.

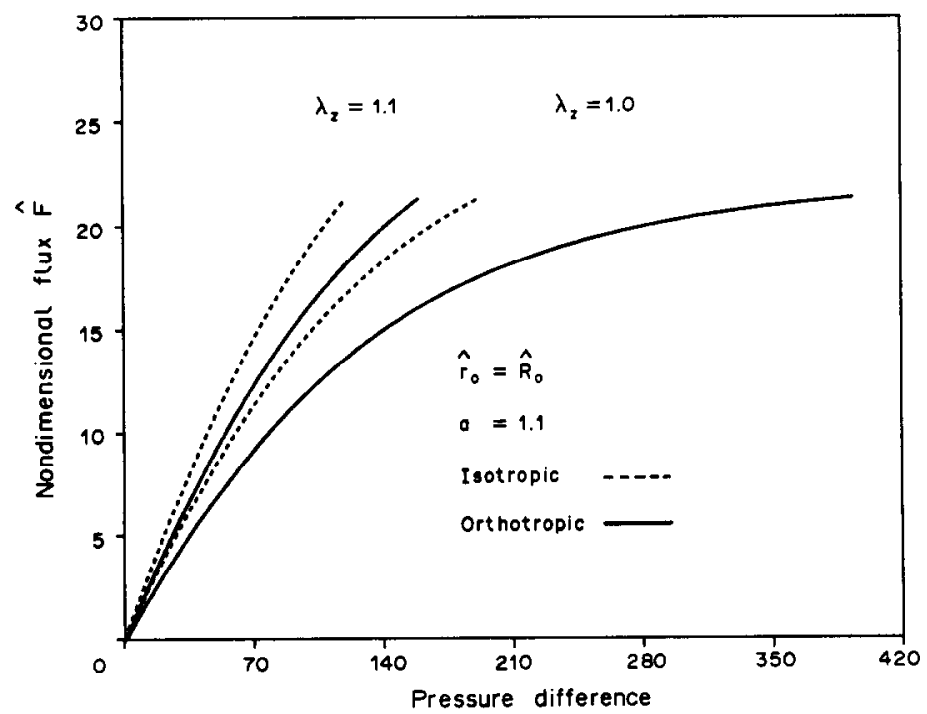

Fig. 5. Effect of the axial deformation on the flux for outward diffusion. 


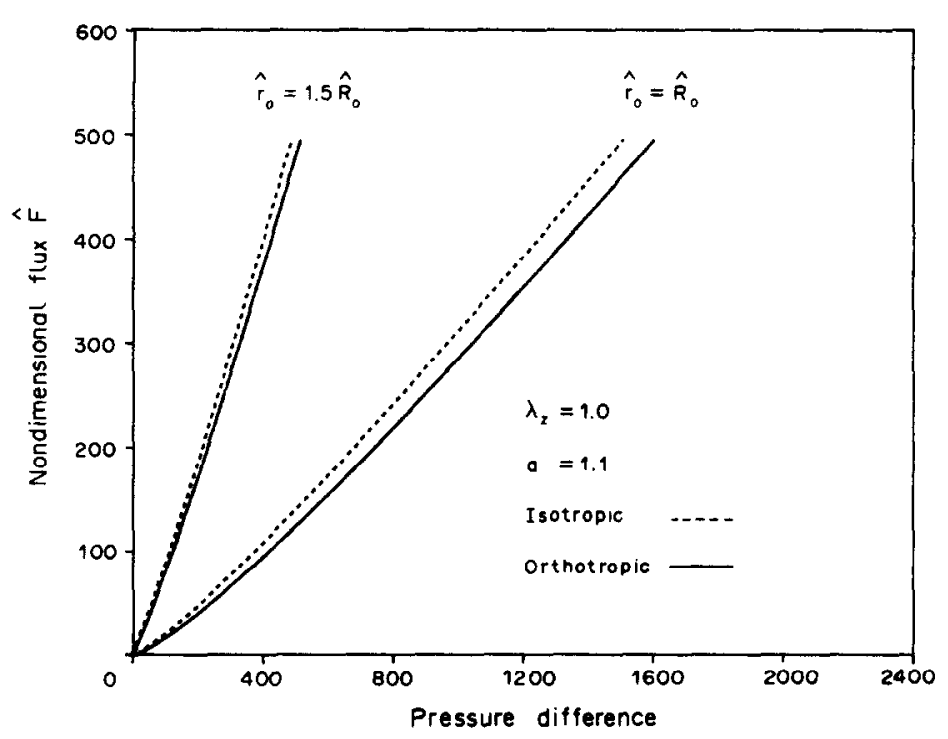

Fig. 6. Effect of the radial deformation on the flux for inward diffusion.

\section{(ii) Effect of the orthotropy of the cylinder on flux}

In Figs 8 and 9, different values are assigned to $K_{1}\left(=2 K_{7}\right)$ and other coefficients being held constant. It can be seen from these figures that the flux is strongly affected by $K_{1}\left(=2 K_{7}\right)$ in both the inward and outward-diffusions. An increase in $K_{1}\left(=2 K_{7}\right)$ results in the decrease of the flux. $K_{2}\left(=2 K_{8}\right)$ and $K_{3}\left(=2 K_{9}\right)$ have relatively weak effect on the inward-diffusion but somewhat stronger influence on the outward-diffusion. Figures 10-13 depict these results.

\section{(iii) Effect of the Helmholtz free energy on flux}

Three different structures of the Helmholtz free energy have been used to study the influence of the structure of the free energy on the diffusion process, as shown in Figs 14 and 15, respectively. One form corresponds to the constitutive expression (48). The other two correspond to subcases which have no linear terms, and only linear terms. The pure linear term case predicts the largest flux and the full-term case expects the smallest flux for both the inward and outward-diffusions.

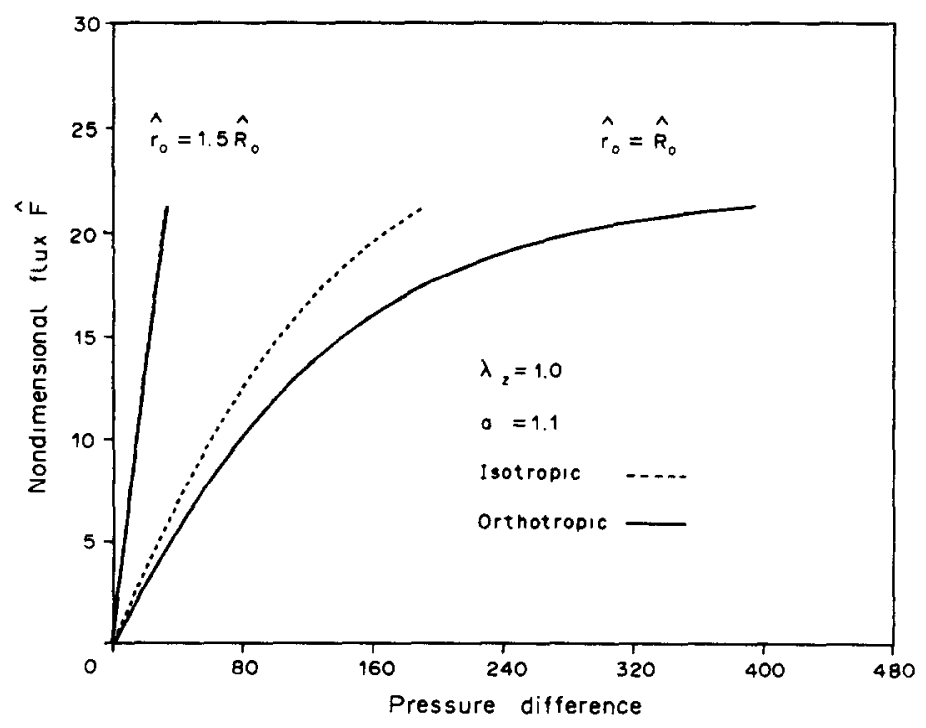

Fig. 7. Effect of the radial deformation on the flux for outward diffusion. 


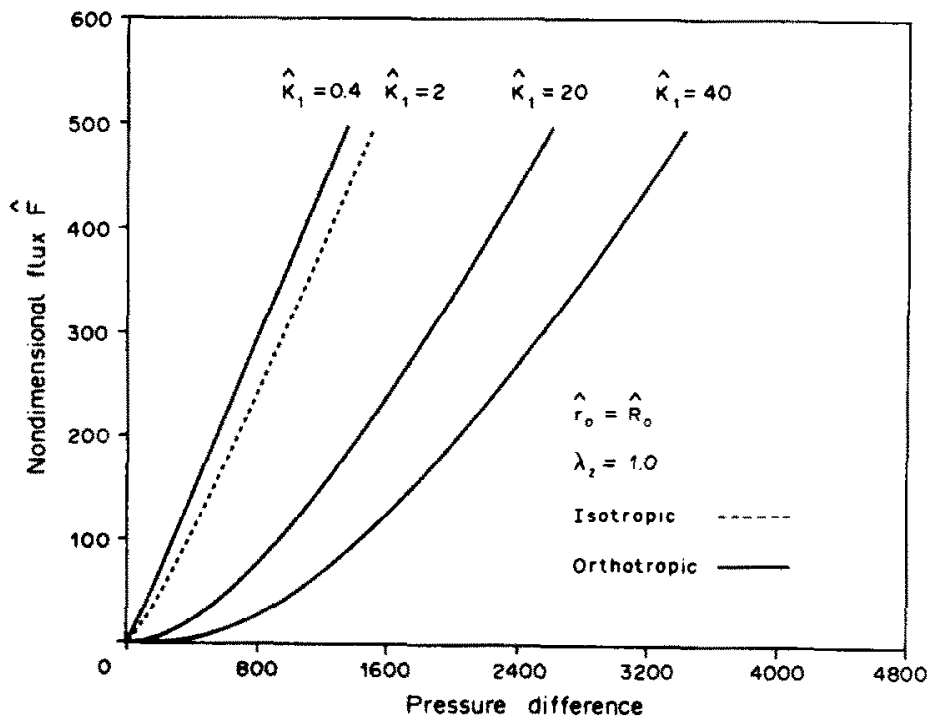

Fig. 8. Effect of the elastic modulus $K_{1}$ on the flux for inward diffusion.

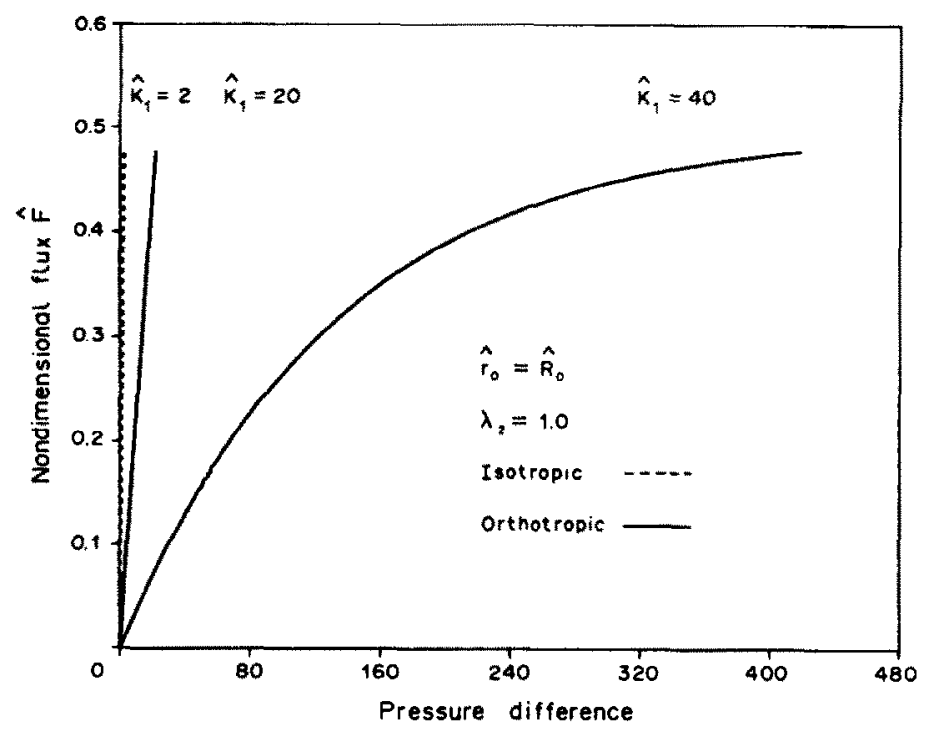

Fig. 9. Effect of the elastic modulus $K_{1}$ on the flux for outward diffusion.

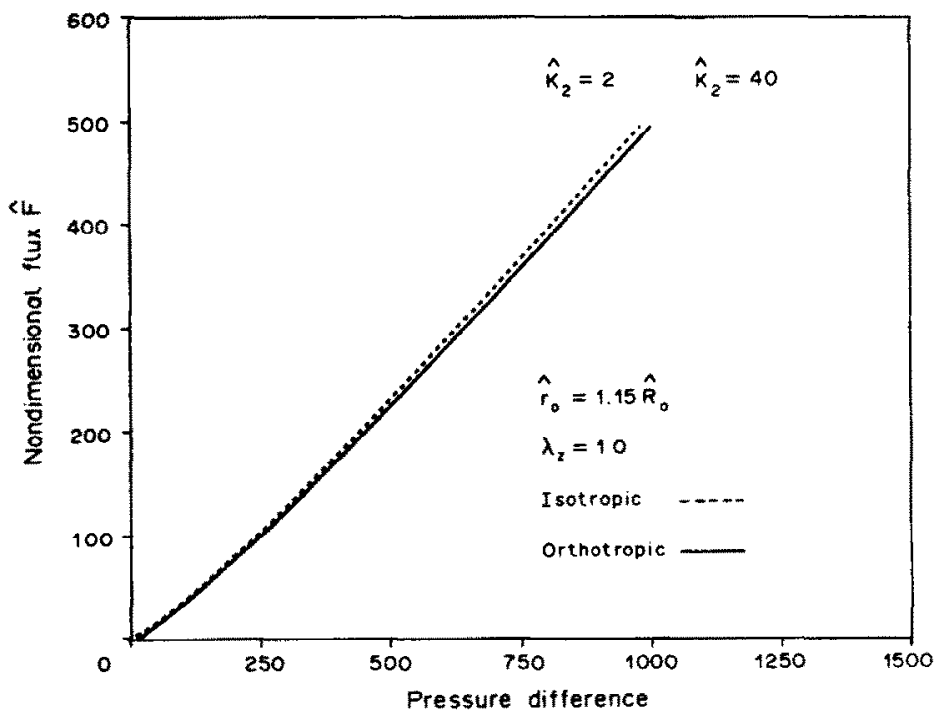

Fig. 10. Effect of the elastic modulus $K_{2}$ on the flux for inward diffusion. 


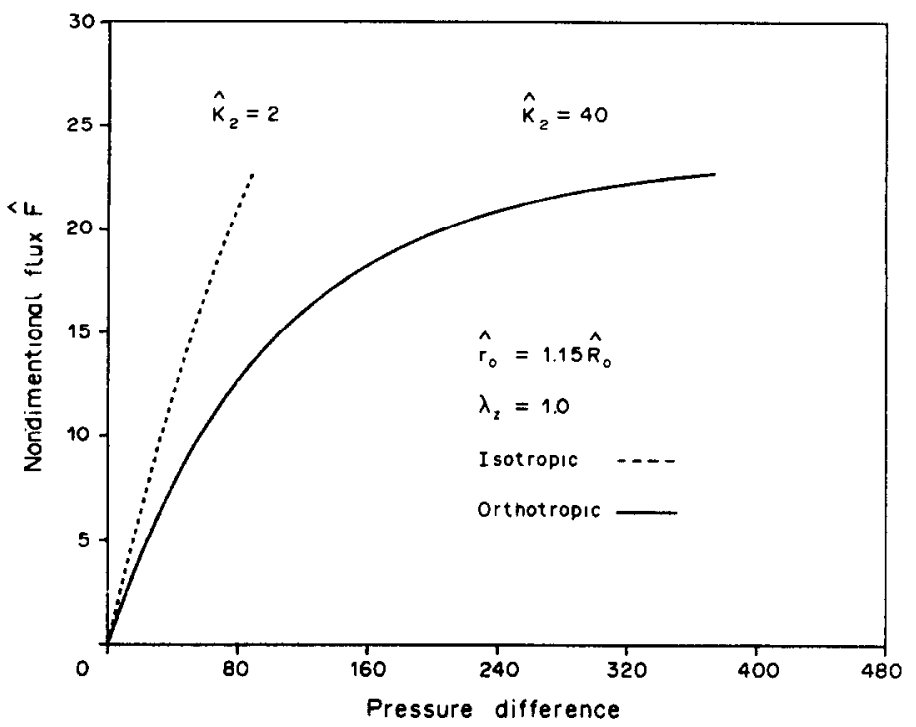

Fig. 11. Effect of the elastic modulus $K_{2}$ on the flux for outward diffusion.

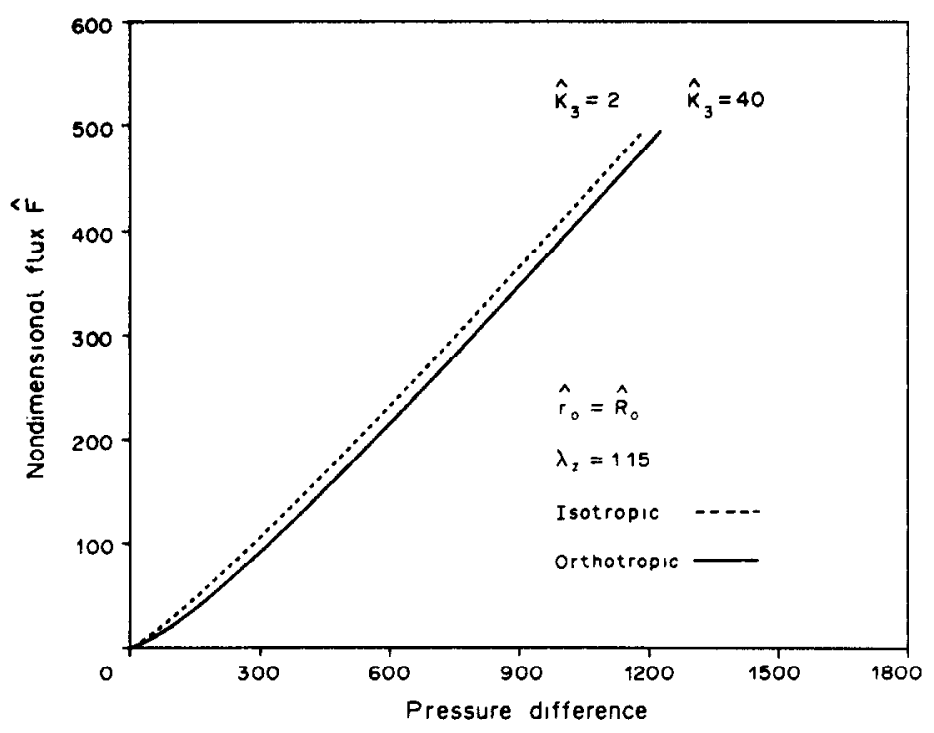

Fig. 12. Effect of the elastic modulus $K_{3}$ on the flux for inward diffusion.

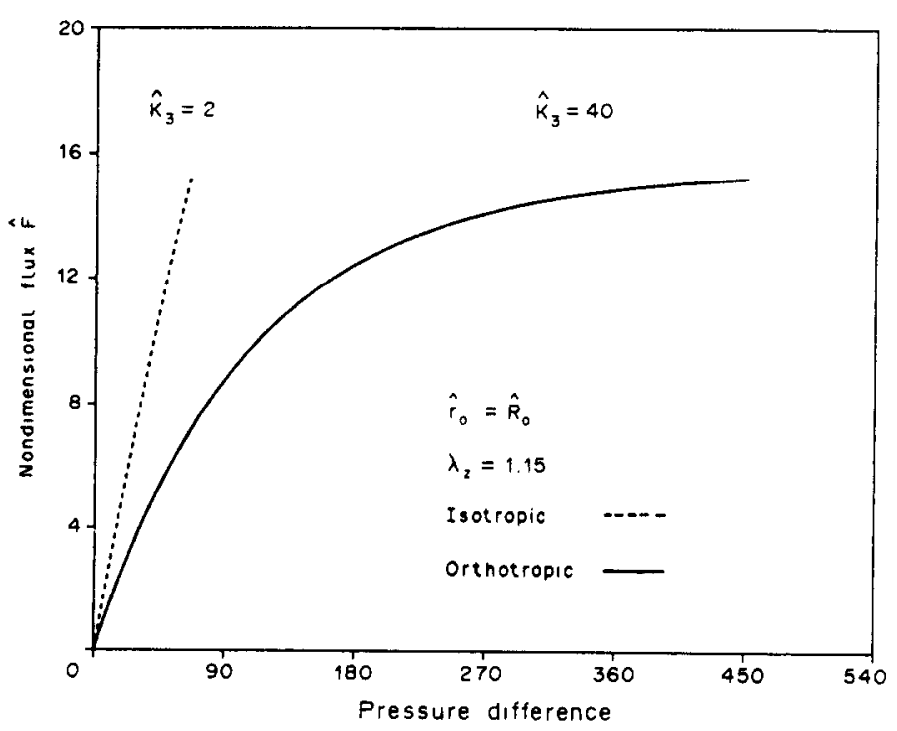

Fig. 13. Effect of the elastic modulus $K_{3}$ on the flux for outward diffusion. 


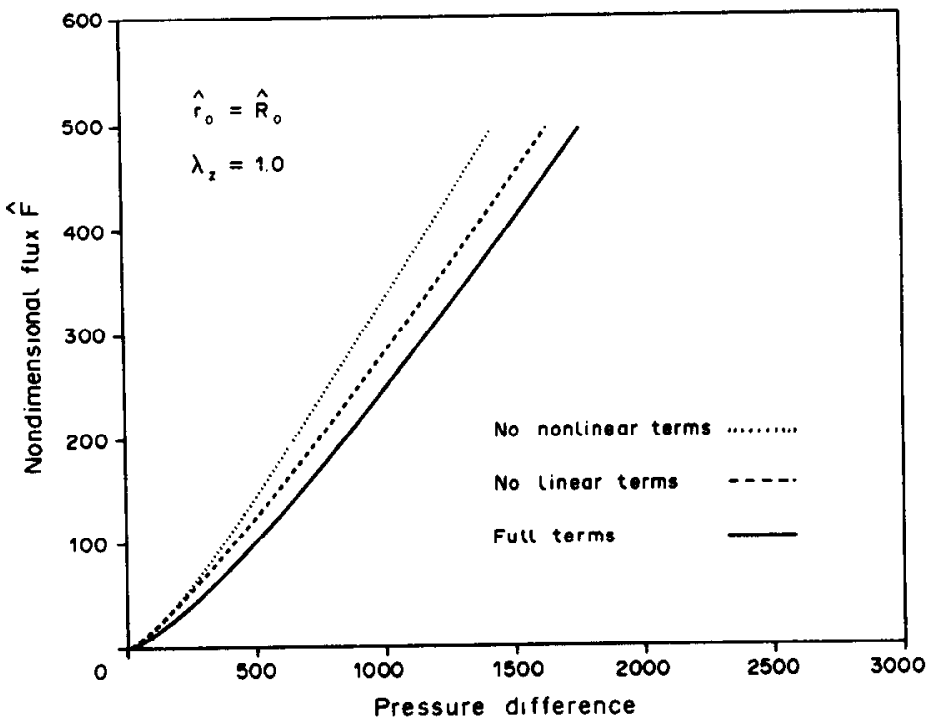

Fig. 14. Effect of the free energy structure on the flux for inward diffusion.

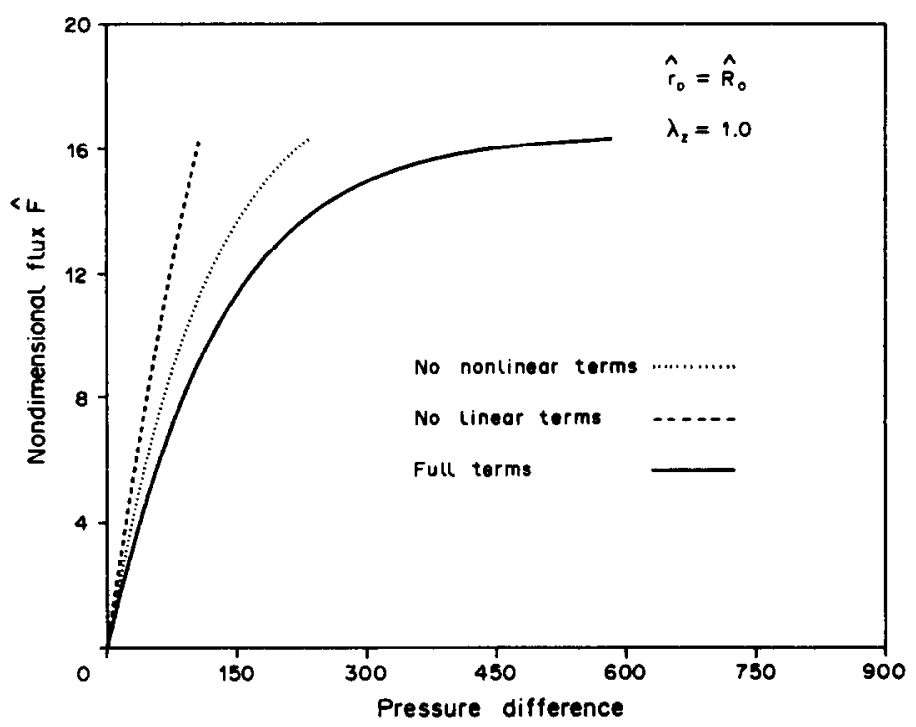

Fig. 15. Effect of the free energy structure on the flux for outward diffusion.

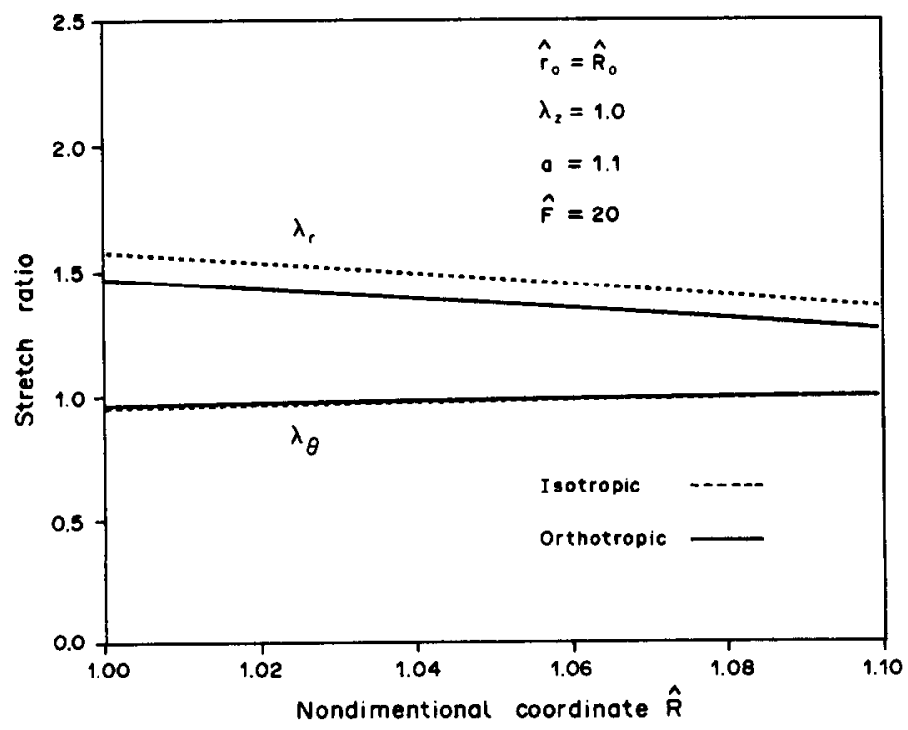

Fig. 16. Variation of the stretch ratios along the cylinder for inward diffusion. 


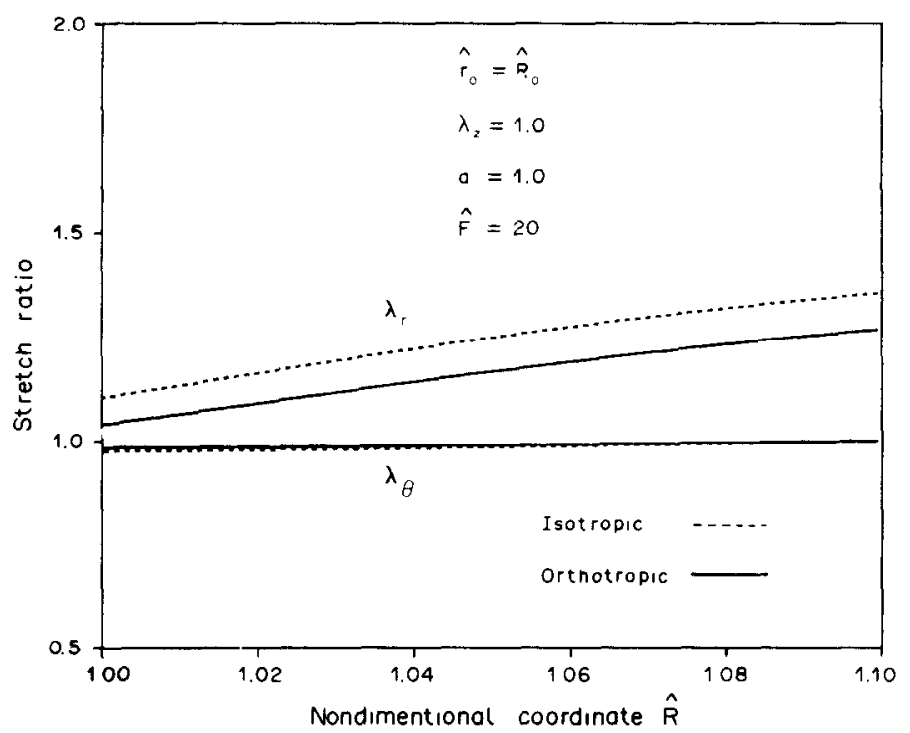

Fig. 17. Variation of the stretch ratios along the cylinder for outward diffusion.

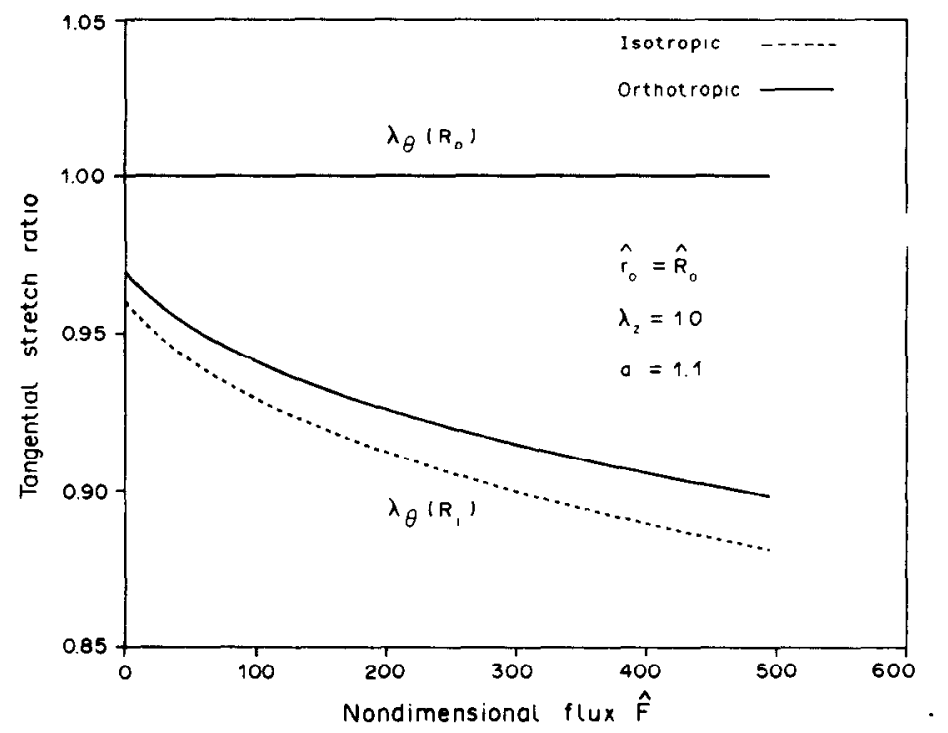

Fig. 18. Variation of the tangential stretch ratio with flux for inward diffusion.

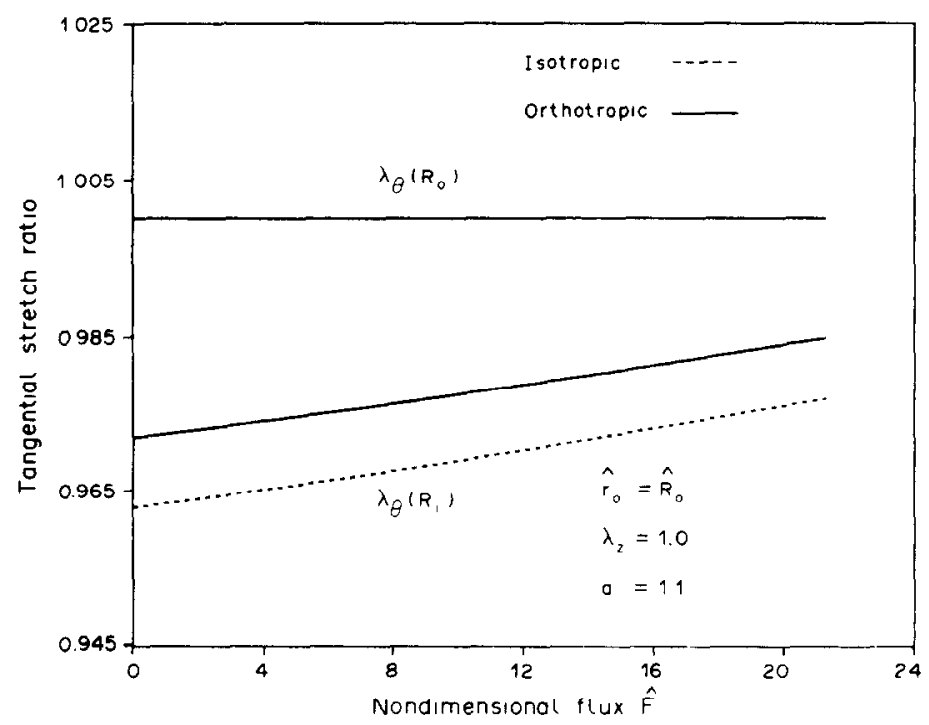

Fig. 19. Variation of the tangential stretch ratio with flux for outward diffusion. 
(iv) Variation of the stretch ratios

Figures 16 and Fig. 17 show how the stretch ratios vary through the cylinder, when the cylinder is thick. The values of $\lambda_{\theta}$ are less than one due to the inward motion of the cylinder caused by swelling. The corresponding values of $\lambda_{\text {r }}$ are relatively large. Figure 18 shows how the radius of the deformed inner surface of the cylinder varies as a function of mass flux for the inward-diffusion. As the flux increases, the amount of swelling increases and the surface moves further inward. A similar conclusion can be made from Fig. 19 for the outward diffusion, but the amount of swelling decreases and the surface moves outward as the flux increases. The influences of the orthotropy of the mixture on the flux $\hat{F}$ and stretch ratios, $\lambda_{\theta}$ and $\lambda_{r}$, can be seen from the above figures.

\section{DIFFUSION OF A FLUID THROUGH A SHEARED AND STRETCHED HOLLOW ELASTIC ORTHOTROPIC CYLINDER}

We shall assume the cylinder has undergone a deformation of the following form in a cylindrical coordinate system:

$$
\begin{gathered}
r=r(R), \\
\theta=\Phi, \\
z=\lambda Z+g(R),
\end{gathered}
$$

as shown in Fig. 20. The flow is assumed to take place in the radial direction only. Then,

$$
w_{r}=w(r) \text { and } w_{\theta}=w_{z}=0 .
$$

The gradient of deformation is

where

$$
\mathbf{F}=\left[\begin{array}{ccc}
\lambda_{r} & 0 & 0 \\
0 & \lambda_{\theta} & 0 \\
g^{\prime} & 0 & \lambda
\end{array}\right]
$$

$$
\lambda_{r}=\frac{\mathrm{d} r}{\mathrm{~d} R}, \quad \lambda_{\theta}=\frac{r}{R} \quad \text { and } \quad g^{\prime}=\frac{\mathrm{d} g}{\mathrm{~d} R}
$$

The Green-St. Venant strain tensor is

$$
\mathbf{E}=\frac{1}{2}\left[\begin{array}{ccc}
\lambda_{r}^{2}+g^{\prime 2}-1 & 0 & \lambda g^{\prime} \\
0 & \lambda_{\theta}^{2}-1 & 0 \\
\lambda g^{\prime} & 0 & \lambda^{2}-1
\end{array}\right]
$$

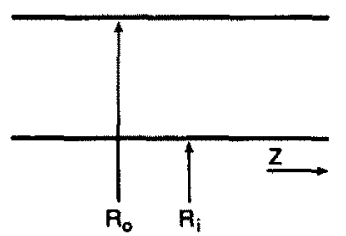

Undelormed State

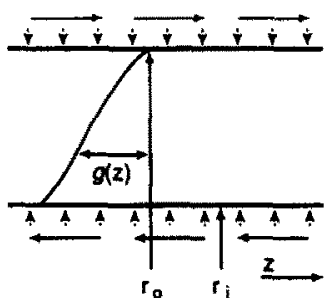

Doformad State

Fig. 20. Diffusion through a sheared cylinder. 
The only non-zero physical components of the gradient of the deformation gradient are

$$
\begin{gathered}
F_{r r ; r}=\frac{\mathrm{d} \lambda_{r}}{\mathrm{~d} r}, \quad F_{r \theta ; \theta}=F_{\theta r ; \theta}=\frac{1}{r}\left(\lambda_{r}-\lambda_{\theta}\right), \\
F_{\theta \theta ; r}=\frac{\mathrm{d} \lambda_{\theta}}{\mathrm{d} r}, \quad F_{z r ; r}=\frac{\mathrm{d} g^{\prime}}{\mathrm{d} r}, \quad F_{z \theta ; \theta}=-\frac{g^{\prime}}{r} .
\end{gathered}
$$

The conservation of mass for the solid and fluid are, respectively

and

$$
\rho_{1} \lambda_{r} \lambda_{\theta} \lambda=\rho_{10}
$$

$$
\frac{1}{r} \frac{\mathrm{d}}{\mathrm{d} r}\left(r \rho_{2} w\right)=0 .
$$

Equation (104) can be integrated to yield

$$
r \rho_{2} w=F=\text { constant, }
$$

where $F$ is the mass flux. It follows from (36) and (37) that the partial stresses are given by

$$
\begin{aligned}
\sigma_{r r}= & -p \frac{\rho_{1}}{\rho_{10}}+\frac{1}{2} \rho \lambda_{r}^{2}\left[2 K_{1}+K_{4}\left(\lambda_{\theta}^{2}-1\right)+K_{5}\left(\lambda^{2}-1\right)+2 K_{7}\left(\lambda_{r}^{2}+g^{\prime 2}-1\right)+2 K_{13} \frac{\rho_{2}}{\rho_{20}}\right] \\
\sigma_{\theta \theta}= & -p \frac{\rho_{1}}{\rho_{10}}+\frac{1}{2} \rho \lambda_{\theta}^{2}\left[2 K_{2}+K_{4}\left(\lambda_{r}^{2}+g^{\prime 2}-1\right)+K_{6}\left(\lambda^{2}-1\right)+2 K_{8}\left(\lambda_{\theta}^{2}-1\right)+2 K_{14} \frac{\rho_{2}}{\rho_{20}}\right] \\
\sigma_{z z}= & -p \frac{\rho_{1}}{\rho_{10}}+\frac{1}{2} \rho\left\{g^{\prime 2}\left[2 K_{1}+K_{4}\left(\lambda_{\theta}^{2}-1\right)+K_{5}\left(\lambda^{2}-1\right)+2 K_{7}\left(\lambda_{r}^{2}+g^{\prime 2}-1\right)+2 K_{13} \frac{\rho_{2}}{\rho_{20}}\right]\right. \\
& \left.+\lambda^{2}\left[2 K_{3}+K_{5}\left(\lambda_{r}^{2}+g^{\prime 2}-1\right)+K_{6}\left(\lambda_{\theta}^{2}-1\right)+2 K_{9}\left(\lambda^{2}-1\right)+2 K_{11} g^{\prime 2}+2 K_{15} \frac{\rho_{2}}{\rho_{20}}\right]\right\} \\
\sigma_{r z}= & \frac{1}{2} \rho \lambda_{r} g^{\prime}\left[2 K_{1}+K_{4}\left(\lambda_{\theta}^{2}-1\right)+K_{5}\left(\lambda^{2}-1\right)+2 K_{7}\left(\lambda_{r}^{2}+g^{\prime 2}-1\right)+K_{11} \lambda^{2}+2 K_{13} \frac{\rho_{2}}{\rho_{20}}\right] \\
\pi_{r r}= & \pi_{\theta \theta}=\pi_{z z}=-p \frac{\rho_{2}}{\rho_{20}}-\frac{1}{2} \rho\left[K_{13}\left(\lambda_{r}^{2}-1\right)+K_{14}\left(\lambda_{\theta}^{2}-1\right)+K_{15}\left(\lambda^{2}-1\right)+2 K f^{\prime}\right] \frac{\rho_{2}}{\rho_{20}}
\end{aligned}
$$

and

$$
\pi_{r \theta}=\pi_{r z}=\pi_{\theta z}=0 .
$$

From (38), the components of the diffusive body force are

$$
\begin{aligned}
b_{r}= & -p \frac{\mathrm{d}}{\mathrm{d} r}\left(\frac{\rho_{1}}{\rho_{10}}\right)-\alpha_{1} \frac{\rho_{1} \rho_{2}}{\rho_{10} \rho_{20}} w+\frac{1}{2} \rho_{1}\left[K_{13}\left(\lambda_{r}^{2}-1\right)+K_{14}\left(\lambda_{\theta}^{2}-1\right)+K_{15}\left(\lambda^{2}-1\right)+2 K f^{\prime}\right] \frac{\mathrm{d}}{\mathrm{d} r}\left(\frac{\rho_{2}}{\rho_{20}}\right) \\
& -\frac{1}{2} \rho_{2}\left\{\left(\lambda_{r} \frac{\mathrm{d} \lambda_{r}}{\mathrm{~d} r}+g^{\prime} \frac{\mathrm{d} g^{\prime}}{\mathrm{d} r}\right)\left[2 K_{1}+K_{4}\left(\lambda_{\theta}^{2}-1\right)+K_{5}\left(\lambda^{2}-1\right)+2 K_{7}\left(\lambda_{r}^{2}+g^{\prime 2}-1\right)+2 K_{13} \frac{\rho_{2}}{\rho_{20}}\right]\right. \\
& \left.+\lambda_{\theta} \frac{\mathrm{d} \lambda_{\theta}}{\mathrm{d} r}\left[2 K_{2}+K_{4}\left(\lambda_{r}^{2}+g^{\prime 2}-1\right)+K_{6}\left(\lambda^{2}-1\right)+2 K_{8}\left(\lambda_{\theta}^{2}-1\right)+2 K_{14} \frac{\rho_{2}}{\rho_{20}}\right]+2 K_{11} \lambda^{2} g^{\prime} \frac{\mathrm{d} g^{\prime}}{\mathrm{d} r}\right\},
\end{aligned}
$$

and

$$
b_{\theta}=b_{z}=0 \text {. }
$$


It follows, from (106)-(110), (113), (114) and (8) on ignoring the inertial terms, that

$$
\begin{aligned}
& -\frac{\rho_{1}}{\rho_{10}} \frac{\partial p}{\partial r}+\alpha \frac{\rho_{1} \rho_{2}}{\rho_{10} \rho_{20}} w-\frac{1}{2} \rho_{1}\left[K_{13}\left(\lambda_{r}^{2}-1\right)+K_{14}\left(\lambda_{\theta}^{2}-1\right)+K_{15}\left(\lambda^{2}-1\right)+2 K f^{\prime}\right] \frac{\mathrm{d}}{\mathrm{d} r}\left(\frac{\rho_{2}}{\rho_{20}}\right) \\
& +\frac{1}{2} \frac{\mathrm{d}}{\mathrm{d} r}\left\{\rho \lambda_{r}^{2}\left[2 K_{1}+K_{4}\left(\lambda_{\theta}^{2}-1\right)+K_{5}\left(\lambda^{2}-1\right)+2 K_{7}\left(\lambda_{r}^{2}+g^{2}-1\right)+2 K_{13} \frac{\rho_{2}}{\rho_{20}}\right]\right\} \\
& +\frac{\rho}{2 r}\left\{\lambda_{r}^{2}\left[2 K_{1}+K_{4}\left(\lambda_{\theta}^{2}-1\right)+K_{5}\left(\lambda^{2}-1\right)+2 K_{7}\left(\lambda_{r}^{2}+g^{\prime 2}-1\right)+2 K_{13} \frac{\rho_{2}}{\rho_{20}}\right]\right. \\
& \left.-\lambda_{\theta}^{2}\left[2 K_{2}+2 K_{4}\left(\lambda_{r}^{2}+y^{\prime 2}-1\right)+K_{6}\left(\lambda^{2}-1\right)+2 K_{8}\left(\lambda_{\theta}^{2}-1\right)+2 K_{14} \frac{\rho_{2}}{\rho_{20}}\right]\right\} \\
& +\frac{1}{2} \rho_{2}\left\{\left(\lambda_{r} \frac{\mathrm{d} \lambda}{\mathrm{d} r}+g^{\prime} \frac{\mathrm{d} g^{\prime}}{\mathrm{d} r}\right)\left[2 K_{1}+K_{4}\left(\lambda_{\theta}^{2}-1\right)+K_{5}\left(\lambda^{2}-1\right)+2 K_{7}\left(\lambda_{r}^{2}+g^{\prime 2}-1\right)+2 K_{13} \frac{\rho_{2}}{\rho_{20}}\right]\right. \\
& +\lambda_{\theta} \frac{\mathrm{d} \lambda_{\theta}}{\mathrm{d} r}\left[2 K_{2}+K_{4}\left(\lambda_{r}^{2}+g^{\prime 2}-1\right)+K_{6}\left(\lambda^{2}-1\right)+K_{8}\left(\lambda_{\theta}^{2}-1\right)+2 K_{14} \frac{\rho_{2}}{\rho_{20}}\right] \\
& \left.+2 K_{11} \lambda^{2} g^{\prime} \frac{\mathrm{d} g^{\prime}}{\mathrm{d} r}\right\}=0 \\
& \frac{1}{2 r} \frac{\mathrm{d}}{\mathrm{d} r}\left\{r \rho \lambda _ { r } g ^ { \prime } \left[2 K_{1}+K_{4}\left(\lambda_{\theta}^{2}-1\right)+K_{5}\left(\lambda^{2}-1\right)\right.\right. \\
& \left.\left.+2 K_{7}\left(\lambda_{r}^{2}+g^{\prime 2}-1\right)+K_{11} \lambda^{2}+2 K_{13} \frac{\rho_{2}}{\rho_{20}}\right]\right\}+\frac{\partial}{\partial z}\left(-p \frac{\rho_{1}}{\rho_{10}}\right)=0 \\
& \frac{1}{r} \frac{\partial}{\partial \theta}\left(-p \frac{\rho_{1}}{\rho_{10}}\right)=0
\end{aligned}
$$

We add (8) and (9), make use of (21) and neglect the inertia of the fluid. The resulting equations are

$$
\begin{aligned}
& -\frac{\partial p}{\partial r}-\frac{1}{2} \frac{\mathrm{d}}{\mathrm{d} r}\left\{\rho\left[K_{13}\left(\lambda_{r}^{2}-1\right)+K_{14}\left(\lambda_{\theta}^{2}-1\right)+K_{15}\left(\lambda^{2}-1\right)+2 K f^{\prime}\right] \frac{\rho_{2}}{\rho_{20}}\right\} \\
& +\frac{1}{2} \frac{\mathrm{d}}{\mathrm{d} r}\left\{\rho \lambda_{r}^{2}\left[2 K_{1}+K_{4}\left(\lambda_{\theta}^{2}-1\right)+K_{5}\left(\lambda^{2}-1\right)+2 K_{7}\left(\lambda_{r}^{2}+g^{\prime 2}-1\right)+2 K_{13} \frac{\rho_{2}}{\rho_{20}}\right]\right\} \\
& +\frac{\rho}{2 r}\left\{\lambda_{r}^{2}\left[2 K_{1}+K_{4}\left(\lambda_{\theta}^{2}-1\right)+K_{5}\left(\lambda^{2}-1\right)+2 K_{7}\left(\lambda_{r}^{2}+g^{\prime 2}-1\right)+2 K_{13} \frac{\rho_{2}}{\rho_{20}}\right]\right. \\
& \left.-\lambda_{\theta}^{2}\left[2 K_{2}+K_{4}\left(\lambda_{r}^{2}+g^{\prime 2}-1\right)+K_{6}\left(\lambda^{2}-1\right)+2 K_{8}\left(\lambda_{\theta}^{2}-1\right)+2 K_{14} \frac{\rho_{2}}{\rho_{20}}\right]\right\}=0
\end{aligned}
$$

$$
\frac{1}{2 r} \frac{\mathrm{d}}{\mathrm{d} r}\left\{r \rho \lambda _ { r } g ^ { \prime } \left[2 K_{1}+K_{4}\left(\lambda_{\theta}^{2}-1\right)+K_{5}\left(\lambda^{2}-1\right)\right.\right.
$$

$$
\left.\left.+2 K_{7}\left(\lambda_{r}^{2}+g^{\prime 2}-1\right)+K_{11} \lambda^{2}+2 K_{13} \frac{\rho_{2}}{\rho_{20}}\right]\right\}+\frac{\partial}{\partial z}(-p)=0
$$

and

$$
\frac{1}{r} \frac{\partial}{\partial \theta}(-p)=0
$$

Notice that (116) and (119) imply

$$
\left(1-\frac{\rho_{1}}{\rho_{10}}\right) \frac{\partial p}{\partial z}=0
$$


By (21), if there is some fluid content in the mixture, i.e. $\rho_{2} \neq 0$, then (121) results in

$$
\frac{\partial p}{\partial z}=0
$$

It follows from (120) and (122) that

$$
p=p(r)
$$

Then, it follows from (119) and (122), after integration, that

$$
\rho \lambda_{r} g^{\prime}\left[2 K_{1}+K_{4}\left(\lambda_{\theta}^{2}-1\right)+K_{5}\left(\lambda^{2}-1\right)+2 K_{7}\left(\lambda_{r}^{2}+g^{\prime 2}-1\right)+K_{11} \lambda^{2}+2 K_{13} \frac{\rho_{2}}{\rho_{20}}\right]=\frac{\text { const. }}{r} \text {. }
$$

From (115) and (118), we have

$$
\begin{aligned}
& -\frac{1}{2} \rho_{1}\left[K_{13}\left(\lambda_{r}^{2}-1\right)+K_{14}\left(\lambda_{\theta}^{2}-1\right)+K_{15}\left(\lambda^{2}-1\right)+2 K f^{\prime}\right] \frac{\mathrm{d}}{\mathrm{d} r}\left(\frac{\rho_{2}}{\rho_{20}}\right) \\
& +\frac{\rho_{1}}{2 \rho_{10}} \frac{\mathrm{d}}{\mathrm{d} r}\left\{\rho\left[K_{13}\left(\lambda_{r}^{2}-1\right)+K_{14}\left(\lambda_{\theta}^{2}-1\right)+K_{15}\left(\lambda^{2}-1\right)+2 K f^{\prime}\right] \frac{\rho_{2}}{\rho_{20}}\right\} \\
& +\alpha \frac{\rho_{1} \rho_{2}}{\rho_{10} \rho_{20}} w+\frac{\rho_{2}}{2 \rho_{20}} \frac{\mathrm{d}}{\mathrm{d} r}\left\{\rho \lambda_{r}^{2}\left[2 K_{1}+K_{4}\left(\lambda_{\theta}^{2}-1\right)+K_{5}\left(\lambda^{2}-1\right)+2 K_{7}\left(\lambda_{r}^{2}+g^{\prime 2}-1\right)+2 K_{13} \frac{\rho_{2}}{\rho_{20}}\right]\right\} \\
& +\frac{\rho}{2 r} \frac{\rho_{2}}{\rho_{20}}\left\{\lambda_{r}^{2}\left[2 K_{1}+K_{4}\left(\lambda_{\theta}^{2}-1\right)+K_{5}\left(\lambda^{2}-1\right)+2 K_{7}\left(\lambda_{r}^{2}+g^{\prime 2}-1\right)+2 K_{13} \frac{\rho_{2}}{\rho_{20}}\right]\right. \\
& \left.-\lambda_{\theta}^{2}\left[2 K_{2}+K_{4}\left(\lambda_{r}^{2}+g^{\prime 2}-1\right)+2 K_{6}\left(\lambda^{2}-1\right)+2 K_{8}\left(\lambda_{\theta}^{2}-1\right)+2 K_{14} \frac{\rho_{2}}{\rho_{20}}\right]\right\} \\
& +\frac{\rho_{2}}{2}\left\{\left(\lambda_{r} \frac{\mathrm{d} \lambda_{r}}{\mathrm{~d} r}+g^{\prime} \frac{\mathrm{d} g^{\prime}}{\mathrm{d} r}\right)\left[2 K_{1}+K_{4}\left(\lambda_{\theta}^{2}-1\right)+K_{5}\left(\lambda^{2}-1\right)+2 K_{7}\left(\lambda_{r}^{2}+g^{\prime 2}-1\right)+2 K_{13} \frac{\rho_{2}}{\rho_{20}}\right]\right. \\
& \left.+\lambda_{\theta} \frac{\mathrm{d} \lambda_{\theta}}{\mathrm{d} r}\left[2 K_{2}+K_{4}\left(\lambda_{r}^{2}+g^{\prime 2}-1\right)+K_{6}\left(\lambda^{2}-1\right)+2 K_{8}\left(\lambda_{\theta}^{2}-1\right)+2 K_{14} \frac{\rho_{2}}{\rho_{20}}\right]+2 K_{11} \lambda^{2} g^{\prime} \frac{\mathrm{d} g^{\prime}}{\mathrm{d} r}\right\}=0
\end{aligned}
$$

Regarding all quantities as functions of the reference coordinate $R$, we have

$$
\begin{aligned}
& -\frac{1}{2} \rho_{1}\left[K_{13}\left(\lambda_{r}^{2}-1\right)+K_{14}\left(\lambda_{\theta}^{2}-1\right)+K_{15}\left(\lambda^{2}-1\right)+2 K f^{\prime}\right] \frac{\mathrm{d}}{\mathrm{d} R}\left(\frac{\rho_{2}}{\rho_{20}}\right) \\
& +\frac{\rho_{1}}{2 \rho_{10}} \frac{\mathrm{d}}{\mathrm{d} R}\left\{\rho\left[K_{13}\left(\lambda_{r}^{2}-1\right)+K_{14}\left(\lambda_{\theta}^{2}-1\right)+K_{15}\left(\lambda^{2}-1\right)+2 K f^{\prime}\right] \frac{\rho_{2}}{\rho_{20}}\right\}+\alpha \frac{\rho_{1} \rho_{2}}{\rho_{10} \rho_{20}} \lambda_{r} w \\
& +\frac{\rho_{2}}{2 \rho_{20}} \frac{\mathrm{d}}{\mathrm{d} R}\left\{\rho \lambda_{r}^{2}\left[2 K_{1}+K_{4}\left(\lambda_{\theta}^{2}-1\right)+K_{5}\left(\lambda^{2}-1\right)+2 K_{7}\left(\lambda_{r}^{2}+g^{\prime 2}-1\right)+2 K_{13} \frac{\rho_{2}}{\rho_{20}}\right]\right\} \\
& +\frac{\rho \lambda_{r}}{2 r} \frac{\rho_{2}}{\rho_{20}}\left\{\lambda_{r}^{2}\left[2 K_{1}+K_{4}\left(\lambda_{\theta}^{2}-1\right)+K_{5}\left(\lambda^{2}-1\right)+2 K_{7}\left(\lambda_{r}^{2}+g^{\prime 2}-1\right)+2 K_{13} \frac{\rho_{2}}{\rho_{20}}\right]\right. \\
& \left.-\lambda_{\theta}^{2}\left[2 K_{2}+K_{4}\left(\lambda_{r}^{2}+g^{\prime 2}-1\right)+K_{6}\left(\lambda^{2}-1\right)+2 K_{8}\left(\lambda_{\theta}^{2}-1\right)+2 K_{14} \frac{\rho_{2}}{\rho_{20}}\right]\right\} \\
& +\frac{1}{2} \rho_{2}\left\{\left(\lambda_{r} \frac{\mathrm{d} \lambda}{\mathrm{d} R}+g^{\prime} \frac{\mathrm{d} g^{\prime}}{\mathrm{d} R}\right)\left[2 K_{1}+K_{4}\left(\lambda_{\theta}^{2}-1\right)+K_{5}\left(\lambda^{2}-1\right)+2 K_{7}\left(\lambda_{r}^{2}+g^{\prime 2}-1\right)+2 K_{13} \frac{\rho_{2}}{\rho_{20}}\right]\right. \\
& +\lambda_{\theta} \frac{\mathrm{d} \lambda_{\theta}}{\mathrm{d} R}\left[2 K_{2}+K_{4}\left(\lambda_{r}^{2}+g^{\prime 2}-1\right)+K_{6}\left(\lambda^{2}-1\right)+2 K_{8}\left(\lambda_{\theta}^{2}-1\right)+2 K_{14} \frac{\rho_{2}}{\rho_{20}}\right] \\
& \left.+2 K_{11} \lambda^{2} g^{\prime} \frac{\mathrm{d} g^{\prime}}{\mathrm{d} R}\right\}=0 .
\end{aligned}
$$

where

$$
“ \prime \prime=\frac{\mathrm{d}}{\mathrm{d} R}, \quad \text { except } \quad f^{\prime}=\frac{\mathrm{d}}{\mathrm{d}\left(\rho_{2} / \rho_{20}\right)} .
$$


Introducing the following non-dimensional qualities

we have

$$
\hat{R}=\frac{R}{R_{i}}, \quad \hat{\rho}_{1}=\frac{\rho_{1}}{\rho_{10}}, \quad \hat{\rho}_{2}=\frac{\rho_{2}}{\rho_{20}}, \quad \hat{\rho}=\frac{\rho}{\rho_{20}},
$$

$$
\begin{aligned}
& -\frac{1}{2} \hat{\rho}_{1} \hat{\rho}_{2}^{\prime}\left[\hat{K}_{13}\left(\lambda_{r}^{2}-1\right)+\hat{K}_{14}\left(\lambda_{\theta}^{2}-1\right)+\hat{K}_{15}\left(\lambda^{2}-1\right)+2 f^{\prime}\right] \frac{\rho_{10}}{\rho_{20}} \\
& +\frac{1}{2} \hat{\rho}_{1}\left\{\hat{\rho} \hat{\rho}_{2}\left[\hat{K}_{13}\left(\lambda_{r}^{2}-1\right)+\hat{K}_{14}\left(\lambda_{\theta}^{2}-1\right)+\hat{K}_{15}\left(\lambda^{2}-1\right)+2 f^{\prime}\right]\right\}^{\prime} \\
& +\frac{\hat{F}}{\hat{R} \lambda_{\theta}^{2} \lambda}+\frac{1}{2} \hat{\rho}_{2}\left\{\hat{\rho} \lambda_{r}^{2}\left[2 \hat{K}_{1}+\hat{K}_{4}\left(\lambda_{\theta}^{2}-1\right)+\hat{K}_{5}\left(\lambda^{2}-1\right)+2 \hat{K}_{7}\left(\lambda_{r}^{2}+g^{\prime 2}-1\right)+2 \hat{K}_{13} \hat{\rho}_{2}\right]\right\}^{\prime} \\
& +\frac{\hat{\rho}_{2} \hat{\rho} \lambda_{r}}{2 \hat{R} \lambda_{\theta}}\left\{\lambda_{r}^{2}\left[2 \hat{K}_{1}+\hat{K}_{4}\left(\lambda_{\theta}^{2}-1\right)+\hat{K}_{5}\left(\lambda^{2}-1\right)+2 \hat{K}_{7}\left(\lambda_{r}^{2}+g^{\prime 2}-1\right)+2 \hat{K}_{13} \hat{\rho}_{2}\right]\right. \\
& \left.-\lambda_{\theta}^{2}\left[2 \hat{K}_{2}+\hat{K}_{4}\left(\lambda_{r}^{2}+g^{\prime 2}-1\right)+\hat{K}_{6}\left(\lambda^{2}-1\right)+2 \hat{K}_{8}\left(\lambda_{\theta}^{2}-1\right)+2 \hat{K}_{14} \hat{\rho}_{2}\right]\right\} \\
& +\frac{1}{2} \hat{\rho}_{2}\left\{\left(\lambda \lambda_{r} \lambda_{r}^{\prime}+g^{\prime} g^{\prime \prime}\right)\left[2 \hat{K}_{1}+\hat{K}_{4}\left(\lambda_{\theta}^{2}-1\right)+\hat{K}_{5}\left(\lambda^{2}-1\right)+2 \hat{K}_{7}\left(\lambda_{r}^{2}+g^{\prime 2}-1\right)+2 \hat{K}_{13} \hat{\rho}_{2}\right]\right. \\
& \left.+\lambda_{\theta} \lambda_{\theta}^{\prime}\left[2 \hat{K}_{2}+\hat{K}_{4}\left(\lambda_{4}^{2}+g^{\prime 2}-1\right)+\hat{K}_{6}\left(\lambda^{2}-1\right)+\hat{K}_{8}\left(\lambda_{\theta}^{2}-1\right)+2 \hat{K}_{14} \hat{\rho}_{2}\right]+2 \hat{K}_{11} \lambda^{2} g^{\prime} g^{\prime \prime}\right\}=0,
\end{aligned}
$$

where

and

$$
\hat{F}=\frac{\alpha_{1} F}{K_{7} \rho_{20}^{2}}, \quad \text { and } \quad \text { ' '”= }=\frac{\mathrm{d}}{\mathrm{d} \hat{R}}, \quad \text { except } f^{\prime}=\frac{\mathrm{d} f}{\mathrm{~d} \hat{\rho}_{2}} \text {, }
$$

$$
\begin{aligned}
& \hat{K}_{1}=\frac{K_{1}}{K}, \quad \hat{K}_{2}=\frac{K_{2}}{K}, \quad \hat{K}_{4}=\frac{K_{4}}{K}, \quad \hat{K}_{5}=\frac{K_{5}}{K}, \\
& \hat{K}_{6}=\frac{K_{6}}{K}, \quad \hat{K}_{7}=\frac{K_{7}}{K}, \quad \hat{K}_{8}=\frac{K_{8}}{K}, \quad \hat{K}_{11}=\frac{K_{11}}{K}, \\
& \hat{K}_{13}=\frac{K_{13}}{K}, \quad \hat{K}_{14}=\frac{K_{14}}{K}, \quad \hat{K}_{15}=\frac{K_{15}}{K} .
\end{aligned}
$$

Differentiating (124) with respect to $R$, we obtain the second governing equation in non-dimensional form

$$
\begin{aligned}
& \left(\hat{R} \hat{\rho} \lambda_{r} \lambda_{\theta} g^{\prime}\right)^{\prime}\left[2 \hat{K}_{1}+\hat{K}_{4}\left(\lambda_{\theta}^{2}-1\right)+\hat{K}_{5}\left(\lambda^{2}-1\right)+2 \hat{K}_{7}\left(\lambda_{r}^{2}+g^{2}-1\right)+\hat{K}_{11} \lambda^{2}+2 \hat{K}_{13} \hat{\rho}_{2}\right] \\
& \quad+\hat{R} \hat{\rho} \lambda_{r} \lambda_{\theta} g^{\prime}\left[2 \hat{K}_{1}+\hat{K}_{4}\left(\lambda_{\theta}^{2}-1\right)+\hat{K}_{5}\left(\lambda^{2}-1\right)+2 \hat{K}_{7}\left(\lambda_{r}^{2}+g^{\prime 2}-1\right)+\hat{K}_{11} \lambda^{2}+2 \hat{K}_{13} \hat{\rho}_{2}\right]^{\prime}=0
\end{aligned}
$$

where the prime means $d / d \hat{R}$. Now we have two governing equations, (129) and (132) for two variables $\hat{r}$ and $\hat{g}$.

Once again, we determine the additional boundary conditions that are necessary for determining the solution to the boundary value problem under consideration. Since there is shearing in this problem, we would have to compute the virtual work due to the shear force and the virtual displacement. Let us denote by suffix $1,2,3$, quantities in the $r, \theta$ and $z$ direction, respectively. In addition to giving virtual displacements $\delta \lambda_{1}, \delta \lambda_{2}$ and $\delta \lambda_{3}$, we will also have to allow for a virtual angular displacement $\delta \alpha$ to the angle of shear $\alpha$. Unlike the previous problem, the basic deformation under consideration also involves shearing. For this problem

$$
\delta A=\frac{\partial A}{\partial E_{11}} \delta E_{11}+\frac{\partial A}{\partial E_{22}} \delta E_{22}+\frac{\partial A}{\partial E_{33}} \delta E_{33}+2 E_{13} \frac{\partial A}{\partial E_{13}^{2}} \delta E_{13}+\frac{\partial A}{\partial \rho_{2}} \delta \rho_{2},
$$

with

$$
\begin{gathered}
\delta E_{11}=\lambda_{1} \delta \lambda_{1}+g^{\prime} \delta g^{\prime}, \\
\delta E_{22}=\lambda_{2} \delta \lambda_{2}, \quad \delta E_{33}=\lambda_{3} \delta \lambda_{3}, \\
\delta E_{13}=\frac{1}{2}\left(g^{\prime} \delta \lambda_{3}+\lambda_{3} \delta g^{\prime}\right) .
\end{gathered}
$$


Thus,

$$
\begin{aligned}
\delta A= & {\left[\lambda_{1} \frac{\partial A}{\partial E_{11}}+\frac{\rho_{20}}{\lambda_{2} \lambda_{3} \lambda_{1}^{2}} \frac{\partial A}{\partial \rho_{2}}\right] \delta \lambda_{1}+\left[\lambda_{2} \frac{\partial A}{\partial E_{22}}+\frac{\rho_{20}}{\lambda_{1} \lambda_{3} \lambda_{2}^{2}} \frac{\partial A}{\partial \rho_{2}}\right] \delta \lambda_{2} } \\
& +\left[\lambda_{3} \frac{\partial A}{\partial E_{33}}+\frac{\rho_{20}}{\lambda_{1} \lambda_{2} \lambda_{3}^{2}} \frac{\partial A}{\partial \rho_{2}}+\frac{1}{2} \lambda_{3} g^{\prime 2} \frac{\partial A}{\partial E_{13}^{2}}\right] \delta \lambda_{3}+\left[g^{\prime} \frac{\partial A}{\partial E_{11}}+\frac{1}{2} \lambda_{3}^{2} g^{\prime} \frac{\partial A}{\partial E_{13}^{2}}\right] \delta g .
\end{aligned}
$$

It can be seen from Fig. 21 that

$$
\begin{aligned}
\delta W= & T_{11}\left(\lambda_{2}+\delta \lambda_{2}\right)\left(\lambda_{3}+\delta \lambda_{3}\right)\left[\left(\lambda_{1}+\delta \lambda_{1}\right) \cos (\alpha+\delta \alpha)-\lambda_{1} \cos \alpha\right] \\
& +T_{22}\left(\lambda_{1}+\delta \lambda_{1}\right)\left(\lambda_{3}+\delta \lambda_{3}\right) \cos \alpha \delta \lambda_{2}+T_{33}\left(\lambda_{1}+\delta \lambda_{1}\right)\left(\lambda_{2}+\delta \lambda_{2}\right) \delta \lambda_{3} \\
& +T_{13}\left(\lambda_{2}+\delta \lambda_{2}\right)\left(\lambda_{3}+\delta \lambda_{3}\right)\left[\left(\lambda_{1}+\delta \lambda_{1}\right) \sin (\alpha+\delta \alpha)-\lambda \sin \alpha\right] .
\end{aligned}
$$

Here $T_{33}$ is not the normal stress acting on the face $z=$ constant but the component of the traction in the $z$-direction on the surface at an angle $\alpha$ such that $\tan \alpha=g^{\prime}$. For the problem where $g^{\prime}=0, T_{33}$ does become the normal stress. We shall assume that $\delta \alpha$ is small enough so that

Also, since

$$
\sin \delta \alpha \approx \delta \alpha \text { and } \cos \delta \alpha \approx 1
$$

$$
\tan \alpha=g^{\prime} \text {, }
$$

it follows that

$$
\delta \alpha=\cos ^{2} \alpha \delta g^{\prime}, \quad \sin ^{2} \alpha=\frac{g^{\prime 2}}{1+g^{\prime 2}}, \quad \cos ^{2} \alpha=\frac{1}{1+g^{\prime 2}} .
$$

Substituting (137)-(139), into (136), we find

$$
\begin{aligned}
\delta W= & \left(T_{11} \cos \alpha+T_{13} \sin \alpha\right) \lambda_{2} \lambda_{3} \delta \lambda_{1}+T_{22} \lambda_{1} \lambda_{3} \cos \alpha \delta \lambda_{2} \\
& +T_{33} \lambda_{1} \lambda_{2} \delta \lambda_{3}+\left(T_{13} \cos \alpha-T_{11} \sin \alpha\right) \lambda_{1} \lambda_{2} \lambda_{3} \cos ^{2} \alpha \delta g^{\prime} .
\end{aligned}
$$

A lengthy but straightforward computation yields

$$
\begin{aligned}
& T_{11}=\left[\rho_{20} A+\rho\left(\rho_{20}-\rho_{2}\right) \frac{\partial A}{\partial \rho_{2}}+\rho \lambda_{1}^{2} \frac{\partial A}{\partial E_{11}}\right] \frac{1}{\sqrt{1+g^{\prime 2}}} \\
& -\rho g^{\prime 2}\left(\frac{\partial A}{\partial E_{11}}+\frac{1}{2} \lambda_{3}^{2} \frac{\partial A}{\partial E_{13}^{2}}\right) \sqrt{1+g^{\prime 2}}, \\
& T_{13}=\left[\rho_{20} A+\rho\left(\rho_{20}-\rho_{2}\right) \frac{\partial A}{\partial \rho_{2}}+\rho \lambda_{1}^{2} \frac{\partial A}{\partial E_{11}}\right] \frac{g^{\prime}}{\sqrt{1+g^{\prime 2}}} \\
& +\rho g^{\prime}\left(\frac{\partial A}{\partial E_{11}}+\frac{1}{2} \lambda_{3}^{2} \frac{\partial A}{\partial E_{13}^{2}}\right) \sqrt{1+g^{\prime 2}} \\
& T_{22}=\left[\rho_{20} A+\rho\left(\rho_{20}-\rho_{2}\right) \frac{\partial A}{\partial \rho_{2}}+\rho \lambda_{2}^{2} \frac{\partial A}{\partial E_{22}^{2}}\right] \sqrt{1+g^{\prime 2}}, \\
& T_{33}=\rho_{20} A+\rho\left(\rho_{20}-\rho_{2}\right) \frac{\partial A}{\partial \rho_{2}}+\rho \lambda_{3}^{2}\left(\frac{\partial A}{\partial E_{33}}+\frac{1}{2} g^{\prime 2} \frac{\partial A}{\partial E_{13}^{2}}\right) \text {. }
\end{aligned}
$$
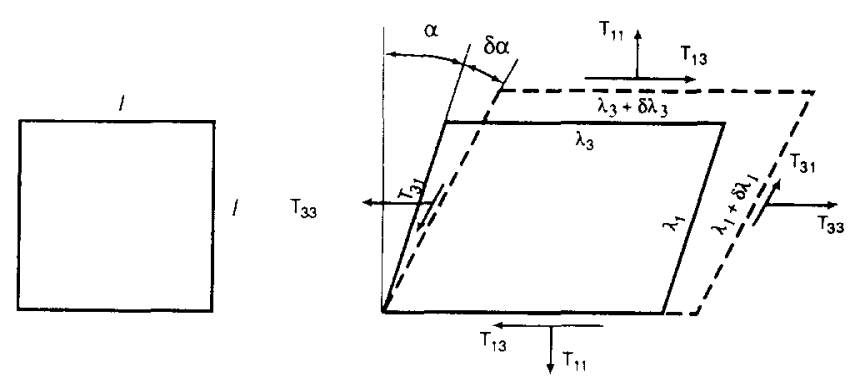

Fig. 21. Variation in the work done due to the variations $\delta \lambda_{1}, \delta \lambda_{2}, \delta \lambda_{3}$ and $\delta \alpha$. 


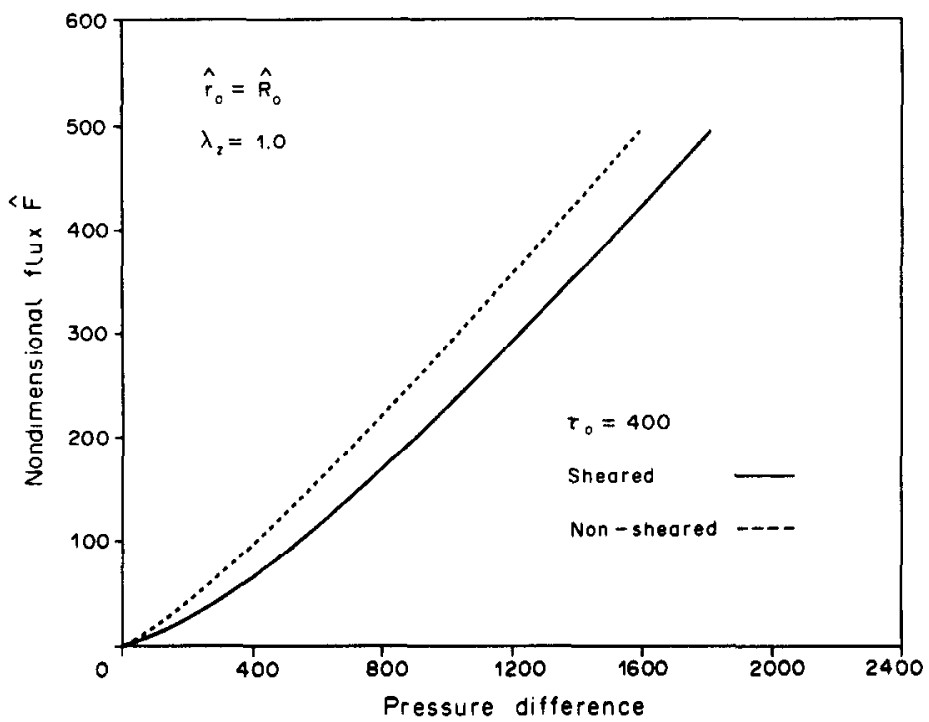

Fig. 22. Effect of shearing on the flux for inward diffusion.

Notice that when $g^{\prime}=0$, the above equations reduce to the additional boundary conditions for the problem studied in the last section. Thus,

$$
\begin{aligned}
-q_{i}= & \left\{\left[\rho_{20} A+\rho\left(\rho_{20}-\rho_{2}\right) \frac{\partial A}{\partial \rho_{2}}+\rho \lambda_{r}^{2} \frac{\partial A}{\partial E_{r r}}\right] \frac{1}{\sqrt{1+g^{\prime 2}}}\right. \\
& \left.-\rho g^{\prime 2}\left(\frac{\partial A}{\partial E_{r r}}+\frac{1}{2} \lambda^{2} \frac{\partial A}{\partial E_{r z}^{2}}\right) \sqrt{1+g^{\prime 2}}\right\}\left.\right|_{R=1} \\
-q_{o}= & \left\{\left[\rho_{20} A+\rho\left(\rho_{20}-\rho_{2}\right) \frac{\partial A}{\partial \rho_{2}}+\rho \lambda_{r}^{2} \frac{\partial A}{\partial E_{r r}}\right] \frac{1}{\sqrt{1+g^{\prime 2}}}\right. \\
& \left.-\rho g^{\prime 2}\left(\frac{\partial A}{\partial E_{r r}}+\frac{1}{2} \lambda^{2} \frac{\partial A}{\partial E_{r z}^{2}}\right) \sqrt{1+g^{\prime 2}}\right\}\left.\right|_{R=a},
\end{aligned}
$$

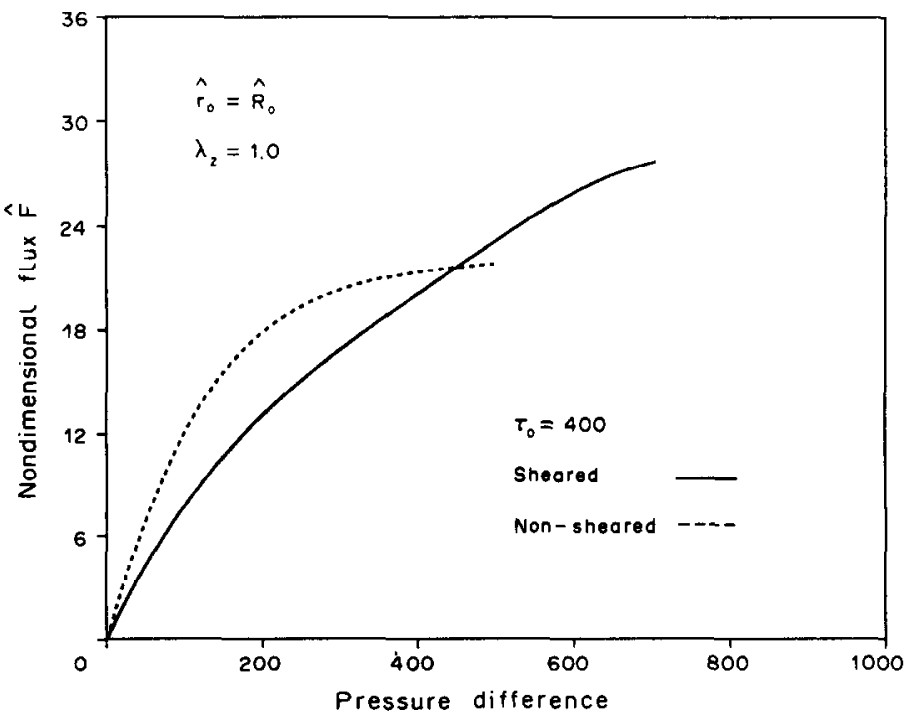

Fig. 23. Effect of shearing on the flux for outward diffusion. 


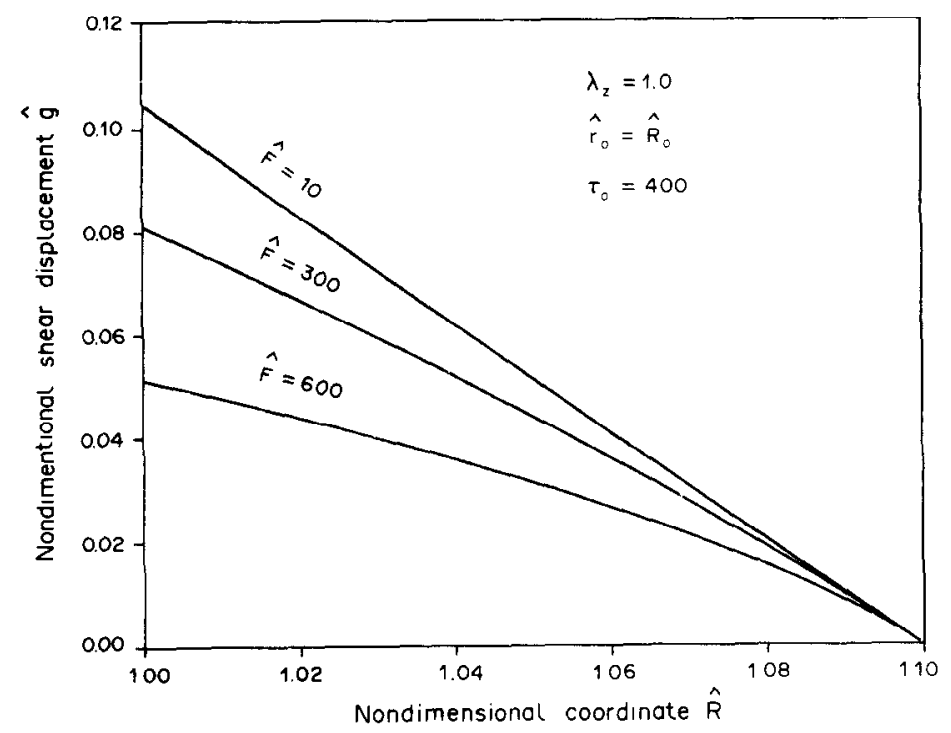

Fig. 24. Variation of the shear displacement along the cylinder thickness for inward diffusion.

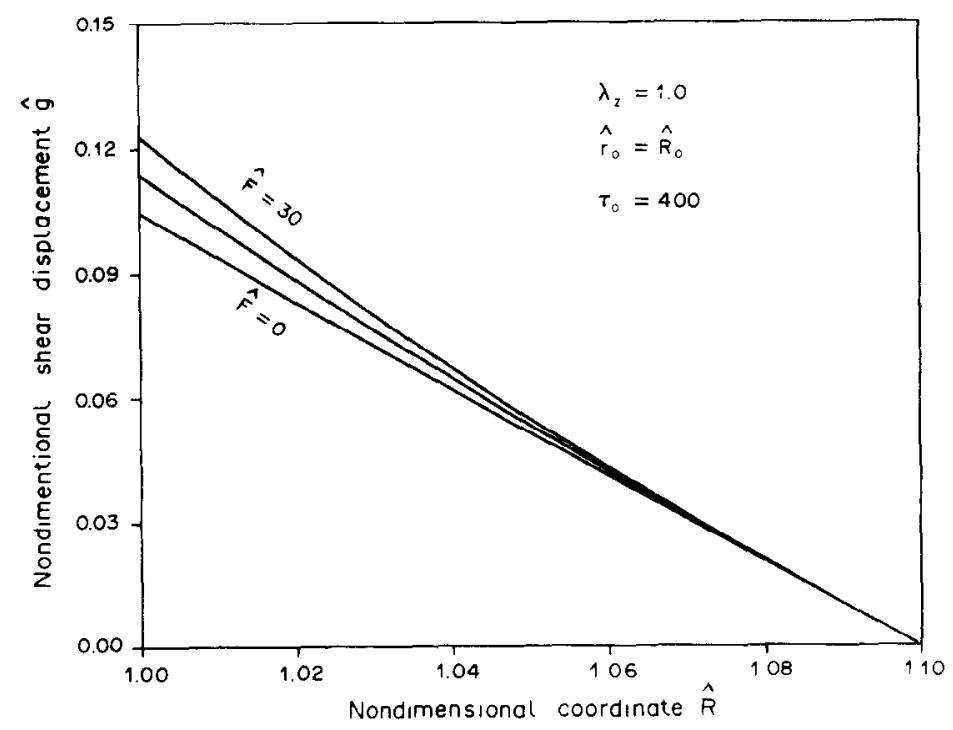

Fig. 25. Variation of the shear displacement along the cylinder thickness for outward diffusion.

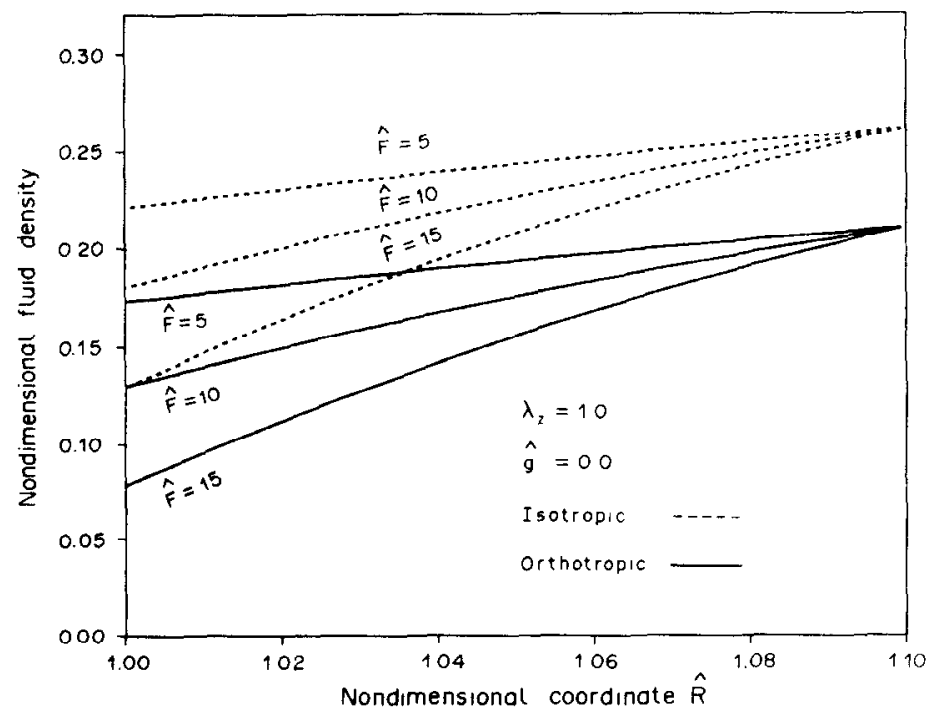

Fig. 26. Variation of the fluid density along the cylinder thickness for inward diffusion. 


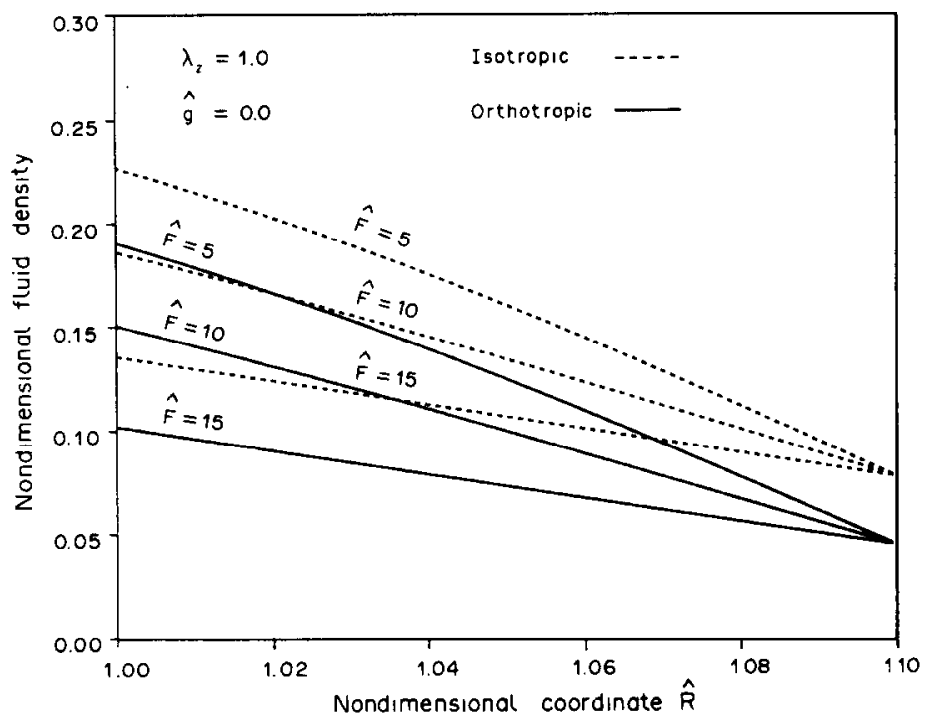

Fig. 27. Variation of the fluid density along the cylinder thickness for outward diffusion.

and

$$
\begin{gathered}
\tau_{0}=\left\{\left[\rho_{20} A+\rho\left(\rho_{20}-\rho_{2}\right) \frac{\partial A}{\partial \rho_{2}}+\rho \lambda_{r}^{2} \frac{\partial A}{\partial E_{r r}}\right] \frac{g^{\prime}}{\sqrt{1+g^{\prime 2}}}\right. \\
\left.+\rho g^{\prime}\left(\frac{\partial A}{\partial E_{r r}}+\frac{1}{2} \lambda^{2} \frac{\partial A}{\partial E_{r z}^{2}}\right) \sqrt{1+g^{\prime 2}}\right\}\left.\right|_{R=a},
\end{gathered}
$$

when $a=R_{o} / R_{i}$ and $q_{i}, q_{o}$ and $\tau_{o}$ denote the pressure on the inner and outer surfaces of the cylinder and the shear stress on the outer surface of the cylinder, respectively.

The method of solving this boundary value problem is essentially the same as was used in the previous section. The values of the material parameters used here are the same as those used in the previous section except $K_{11}=830$. The subroutine and error tolerance are also the same. We discuss the effect of shearing on the diffusions. Figures 22 and 23 show that the shear displacement of the cylinder decreases the mass flux for any value of the pressure difference for both of the inward and outward-diffusions. Shearing tends to decrease the mass flux. The variation of the shear displacement $\hat{g}$ through the cylinder with the mass flux under the fixed shear stress, $\tau_{0}=400$, are depicted in Figs 24 and 25, respectively. Note that the deformation of the mixture is highly non-homogeneous. Under a fixed shear stress on boundary, increasing the mass flux decreases shear displacement for the inward-diffusion and increases the displacement for the outward-diffusion. Figures 26 and 27 show how the fluid dispersed through the cylinder for the inward and the outward-diffusion, respectively.

\section{REFERENCES}

[1] C. TRUESDELL, Rend. Lincei 22, 33-38 (1957).

2] R. M. BOWEN, Continuum Physics (Edited by A. C. ERINGEN), Vol. III. Academic Press, New York (1975).

[3] R. J. ATKIN and R. E. CRAINE, Q. J. Mech. Appl. Math. 29, Pt 2 (1976).

[4] A. BEDFORD and D. S. DRUMHELLER, Int. J. Engng Sci. 21, 863-960 (1983).

[5] S. PASSMAN, J. NUNZIATO and E. K. WALSH, A theory of multiphase mixtures. Sandia Report, SAND82-2261 (1983).

[6] J. J. SHI, K. R. RAJAGOPAL and A. S. WINEMAN, Int. J. Engng Sci. 9, 871-889 (1981).

[7] $\mathrm{K}$ : R. RAJAGOPAL, J. J. SHI and A. S. WINEMAN, Int. J. Engng Sci. 21, 1171-1183 (1983).

[8] K. R. RAJAGOPAL, A. S. WINEMAN and M. GANDHI, Int. J. Engng Sci. 24, 1453-1463 (1986).

[9] M. GANDHI, K. R. RAJAGOPAL and A. S. WINEMAN, Int. J. Engng Sci. 25, 1441-1457 (1987).

[10] A. E. GREEN and P. M. NAGHDI, Q. J. Mech. Appl. Math. 22, 427-438 (1969).

11] N. MILLS, Int. J. Engng Sci. 4, 97-112 (1966).

[12] A. E. GREEN and J. E. ADKINS, Large Elastic Deformation. Oxford University Press (1970).

[13] F. DAI, Diffusion of fluids through anisotropic solids. Dissertation, University of Pittsburgh, (1989).

[14] D. R. PAUL and O. M. EBRA-LIMA, J. Appl. Polymer Sci. 14, 2201-2224 (1970). 